\title{
Comparing supervised and unsupervised approaches to multimodal emotion recognition
}

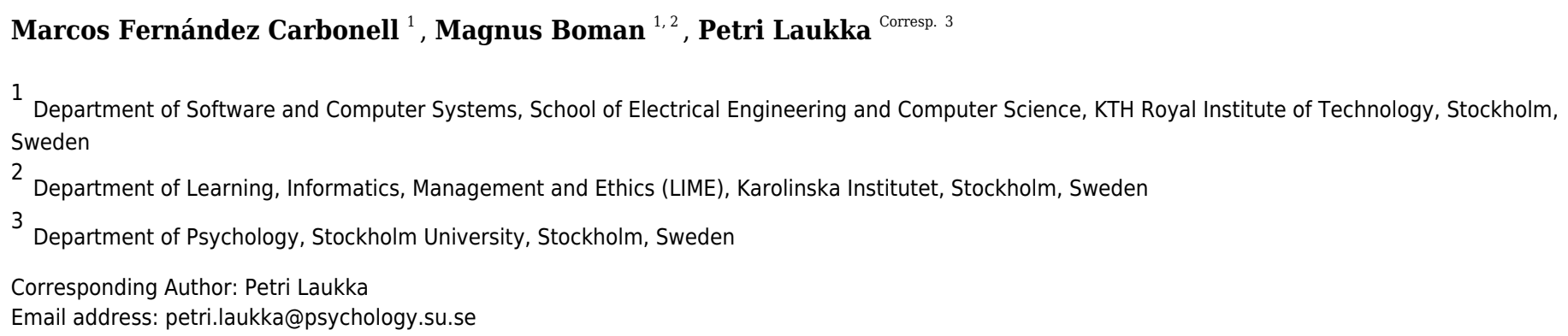

We investigated emotion classification from brief video recordings from the GEMEP database wherein actors portrayed 18 emotions. Vocal features consisted of acoustic parameters related to frequency, intensity, spectral distribution, and durations. Facial features consisted of facial action units. We first performed a series of person-independent supervised classification experiments. Best performance ( $A U C=0.88$ ) was obtained by merging the output from the best unimodal vocal (Elastic Net, $A U C=0.82$ ) and facial (Random Forest, $A \cup C=0.80$ ) classifiers using a late fusion approach and the product rule method. All 18 emotions were recognized with above-chance recall, although recognition rates varied widely across emotions (e.g., high for amusement, anger, and disgust; and low for shame). Multimodal feature patterns for each emotion are described in terms of the vocal and facial features that contributed most to classifier performance. Next, a series of exploratory unsupervised classification experiments were performed to gain more insight into how emotion expressions are organized. Solutions from traditional clustering techniques were interpreted using decision trees in order to explore which features underlie clustering. Another approach utilized various dimensionality reduction techniques paired with inspection of data visualizations. Unsupervised methods did not cluster stimuli in terms of emotion categories, but several explanatory patterns were observed. Some could be interpreted in terms of valence and arousal, but actor and gender specific aspects also contributed to clustering. Identifying explanatory patterns holds great potential as a meta-heuristic when unsupervised methods are used in complex classification tasks. 
3 Comparing supervised and unsupervised approaches to multimodal emotion recognition

$6{ }^{1}$ Department of Software and Computer Systems, School of Electrical Engineering and

7 Computer Science, KTH Royal Institute of Technology, Stockholm, Sweden

${ }^{2}$ Department of Learning, Informatics, Management and Ethics (LIME), Karolinska Institutet, 10

11

Stockholm, Sweden

${ }^{2}$ Department of Psychology, Stockholm University, Stockholm, Sweden

15 Running title: Multimodal emotion recognition

Word count: 5664

17 Number of tables: 4

18 Number of figures: 9 (+ 10 supplementary figures)

21 Correspondence:

22 Petri Laukka

23 Stockholm University, Department of Psychology

2410691 Stockholm, Sweden

25 e-mail: petri.laukka@psychology.su.se 


\section{Abstract}

We investigated emotion classification from brief video recordings from the GEMEP database wherein actors portrayed 18 emotions. Vocal features consisted of acoustic parameters related to frequency, intensity, spectral distribution, and durations. Facial features consisted of facial action units. We first performed a series of person-independent supervised classification experiments. Best performance $(\mathrm{AUC}=0.88)$ was obtained by merging the output from the best unimodal vocal (Elastic Net, AUC $=0.82)$ and facial (Random Forest, $A U C=0.80$ ) classifiers using a late fusion approach and the product rule method. All 18 emotions were recognized with abovechance recall, although recognition rates varied widely across emotions (e.g., high for amusement, anger, and disgust; and low for shame). Multimodal feature patterns for each emotion are described in terms of the vocal and facial features that contributed most to classifier performance. Next, a series of exploratory unsupervised classification experiments were performed to gain more insight into how emotion expressions are organized. Solutions from traditional clustering techniques were interpreted using decision trees in order to explore which features underlie clustering. Another approach utilized various dimensionality reduction techniques paired with inspection of data visualizations. Unsupervised methods did not cluster stimuli in terms of emotion categories, but several explanatory patterns were observed. Some could be interpreted in terms of valence and arousal, but actor and gender specific aspects also contributed to clustering. Identifying explanatory patterns holds great potential as a metaheuristic when unsupervised methods are used in complex classification tasks. 
Comparing supervised and unsupervised approaches to multimodal emotion recognition

\section{Introduction}

56

Meta-analyses of emotion perception studies suggest that human judges are able to accurately infer emotions from nonverbal vocal and facial behavior, also in cross-cultural settings (e.g., Elfenbein \& Ambady, 2002; Laukka \& Elfenbein, 2021). However, it has proved more difficult 
73 to define the physical features reliably associated with specific emotions. Juslin and Laukka

74 (2003) proposed that nonverbal communication of emotion through the voice is based on a

75 number of probabilistic and partly redundant acoustic cues. Probabilistic cues are not perfect

76 indicators of the expressed emotion because they are not always associated with that emotion and

77 can also be used in the same way to express different emotions. For example, high mean

78 fundamental frequency (F0) can be associated with both happiness and fear. Several partly

79 redundant cues can, in turn, be associated with the same emotion. For example, anger can be

80 associated with high levels of both voice intensity and high-frequency energy. Barrett et al.

81 (2019) similarly noted that facial cues (e.g., smiles) are only probabilistically associated with any

82 one emotion (e.g., happiness), and that similar configurations of facial movements can be

83 associated with more than one emotion.

84

85 The combination of probabilistic and partly redundant cues entails that there may be several cue

86 combinations that are associated with the same emotion, which leads to a robust and flexible

87 system of communication (Juslin \& Laukka, 2003). For example, Srinivasan and Martinez

88 (2018) recently reported that several different facial configurations were used to communicate

89 the same emotion in naturalistic settings (e.g., they reported 17 different configurations for

90 happiness). Machine learning methods are increasingly used to detect patterns in this type of

91 high-dimensional probabilistic data. Recent years have seen much activity in the field of

92 machine-based classification of emotions from facial (e.g., Li \& Deng, 2020) and vocal (e.g.,

93 Schuller, 2018) expressions. Classifiers often perform on par with human judges, although

94 performance also varies across emotions and databases (Krumhuber et al., 2021b). The majority

95 of classification studies have been performed on unimodal stimuli (either vocal or facial), but 
96

97

98

combining features from several modalities has been shown to increase classification accuracy (see D’Mello \& Kory, 2015, for a meta-analysis). The number of multimodal classification studies is steadily increasing (Poria et al., 2017), and recent studies explore a wide variety of approaches (e.g., Bhattacharya et al., 2021; Lingenfelser et al., 2018; Mai et al., 2020; Siriwardhana et al., 2020; Tzirakis et al., 2017; Wang et al., 2020).

In the current study, we first compare how unimodal and multimodal classifiers perform in the classification of 18 different emotions from brief video recordings. Recordings are taken from the Geneva Multimodal Emotion Portrayal (GEMEP) corpus (Bänziger et al., 2012), which contains dynamic audio-video emotion expressions portrayed by professional actors. This approach extends most previous classification studies which have focused on a much smaller number of emotions, but is in line with recent perception studies which suggest that human judges can perceive a wide variety of emotions (e.g., Cordaro et al., 2018). Different actors are used for training and testing in all classification experiments, to avoid person bias. We also contribute by providing details of which features are important for classification of which emotions-something only rarely done in machine classification (see Krumhuber et al., 2021b, for a recent example).

We analyze the physical properties of vocal expressions by extracting the features included in the Geneva Minimal Acoustic Parameter Set (GeMAPS; Eyben et al., 2016). This parameter set is commonly used in affective computing and provides features related to the frequency, intensity, spectral energy, and temporal characteristics of the voice. Facial Action Units (AUs) (Ekman \& Friesen, 1978)-which is one of the most comprehensive and objective ways to describe facial expressions (Martinez et al., 2019)-are also extracted. This selection of vocal and facial features 
120 allows for comparisons with the previous literature on emotion expression. Finally, we compare

121 the results from the supervised classification experiments with results from unsupervised

122 classification. Unsupervised methods may reveal new information about how emotion

123 expressions are organized, because they are not restricted to any pre-defined emotion categories

124 (e.g., Azari et al., 2020).

125

126

\section{Methods}

127 Data, code, and additional computational information are openly available on GitHub (see Data 128 Availability statement).

\section{Emotion expressions}

131 The emotion expressions used in this study were taken from the GEMEP database (Bänziger et 132 al., 2012) and consist of 1,260 video files in which ten professional actors, coached by a 133 professional director, convey 18 affective states. They do this by uttering two different

134 pseudolinguistic phoneme sequences, or a sustained vowel 'aaa'. The emotions portrayed in this 135 dataset are: admiration, amusement, anger, anxiety/worry, contempt, despair, disgust, interest, 136 irritation, joy/elation, panic fear, sensual pleasure, pride, relief, sadness, shame, surprise, and 137 tenderness. The number of files per emotion and actor can be seen in Figure S1. The GEMEP 138 dataset was chosen for its high naturalness ratings and wide range of included emotions. It is 139 widely used in classification studies (e.g., Schuller et al., 2019; Valstar et al., 2012), although 140 few previous studies have included all 18 emotions.

\section{Feature extraction and pre-processing}


143 Audio features were obtained using openSMILE 2.3.0 (Eyben et al., 2013), an open-source

144 toolkit that allows for the extraction of a wide variety of parameter sets. In this study, two

145 different versions of the GeMAPS (Geneva Minimalistic Acoustic Parameter Set) (Eyben et al.,

146 2016) were evaluated. While GeMAPS contains 62 non-time series parameters with prosodic,

147 excitation, vocal tract, and spectral descriptors, the extended version eGeMAPS adds a small set

148 of cepstral descriptors, reaching a total of 88 features.

150 Video features were obtained using OpenFace 2.2.0 (Baltrušaitis et al., 2018), an open-source

151 facial behavior analysis toolkit. OpenFace offers an extensive range of parameters such as facial

152 landmark detection, head pose estimation, and eye-gaze estimation. However, our study focused

153 on Facial Action Units (AUs) (Ekman \& Friesen, 1977) and the toolkit provides the intensity of

15417 AUs per frame (i.e., 1, 2, 4, 5, 6, 7, 9, 10, 12, 14, 15, 17, 20, 23, 25, 26, \& 45). AU detection

155 is based on pre-trained models for the detection of facial landmarks, and uses dimensionality-

156 reduced histograms of oriented gradients (HOGs) from face image and facial shape features in

157 Support Vector Machine analyses (for details, see Baltrušaitis et al., 2015; 2018).

158

159 We removed instances where AU detection was deemed unreliable. The OpenFace toolkit

160 provides two indicators per instance that aided the data cleaning process: the confidence and

161 success rates. The former refers to how reliable the extracted features are (continuous value from

162 zero to one), whereas the latter denotes if the facial tracking is favorable or not (binary value).

163 Taking this into consideration, instances with a confidence rate lower than 0.98 or an

164 unfavorable success rate were dropped. Ninety percent of instances received a confidence rating

165 of 0.98 or higher (see Figure S2 for more details). The percentage of instances with unfavorable 
166 success rate was very low (0.58\%). In total, the number of instances decreased by $9.94 \%$ after

167 the cleaning process and caused the deletion of one entire file. Two steps were followed to

168 achieve data consistency. First, the corresponding audio track was deleted. Secondly, video

169 instances were grouped by file and the framewise feature intensity scores from OpenFace were

170 used to compute the following functionals for each AU: arithmetic mean; coefficient of

171 variation; 20th, 50th, and 80th percentile; percentile range (20th to 80th percentile); and the

172 number of peaks (using the mean value as an adaptive threshold). Lastly, data was normalized

173 using min-max normalization.

174

After cleaning, data from both modalities was prepared in the following ways. For the supervised

176 approach, data was randomly assembled in five groups ensuring that all stimuli were represented

177 and that actors in the training set were not included in the validation set. This grouping strategy

178 resulted in different pairs of actors (one female-female, one male-male, and three female-male

179 pairs) which facilitated the later use of LOGO CV (Leave-One-Group-Out Cross-Validation).

180 For the unsupervised approach, the normalized feature vectors from both modalities were

181 concatenated as in an early fusion scenario (Wöllmer et al., 2013), yielding a dataset with 207

182 dimensions and 1,259 observations.

Experiments

185 Supervised learning. We evaluated different multimodal late and early fusion pipelines (see 186 Atrey et al., 2010; Dong et al., 2015), and compared them with the best audio and video unimodal classifiers. After this, the relations between emotion categories and audiovisual cues were investigated. The multimodal pipelines utilize machine learning algorithms such as Linear 
189 Classifiers with Elastic Net regularization, k-NN, Decision Tree, and Random Forest. The first

190 three were used since they are some of the most commonly employed methods for emotion

191 recognition (Marechal et al., 2019), whereas Random Forest was used because it is known as one

192 of the best out-of-the-box classifiers (Sjardin et al., 2016). We will use the term 'Elastic Net

193 classifiers' to refer to linear classifiers with Elastic Net regularization.

194

195

196

197

198

199

200

201

202

203

204

205

206

207

208

209

210
Late fusion. This approach can be summarized into three steps. First, audio and video classifiers were separately subjected to a modeling and selection process. Second, different techniques were tested for fusing the outputs of the audio and video classifiers. Third, the best late fusion pipeline was compared to the best unimodal classifiers. Note that the best unimodal classifiers correspond to the strongest models, in terms of their validation Area Under the Curve (AUC) (see Jeni et al., 2013), picked in the first step. Next, a more in-depth description of the previously stated stages is given. The first step can be split, in turn, into two phases, repeated for each modality. First, LOGO CV was employed for hyperparameter tuning over the dataset.

Second, once the best parameters for each classifier (Elastic Net, k-NN, Decision Tree, and Random Forest) were found, the validation AUC was used to choose between types of machine learning classifiers. The second step followed the same process as the previous one but evaluating different fusion methods, such as the maximum rule, sum rule, product rule, weight criterion, rule-based, and model-based (Elastic Net, k-NN, and Decision Tree) methods (Atrey et al., 2010; Dong et al., 2015). The last step consisted of comparing the best late fusion pipeline to the best unimodal classifiers in terms of their validation AUC. 

instances were carefully concatenated. Second, different types of machine learning classifiers were subjected to a modeling and selection process. Third, the best early fusion pipeline was compared to the best unimodal classifiers. In more detail, the first step joins audio and video hyperparameter tuning over the dataset, and then the best parameters for each classifier (Elastic

217 Net, k-NN, Decision Tree, and Random Forest) were obtained. The validation AUC was used to choose between types of machine learning classifiers. The third and final step compared the best early fusion pipeline to the best unimodal classifiers in terms of their validation AUC.

Unsupervised learning. In order to find meaningful patterns in the multimodal data, two different paths were taken. On the one hand, a more traditional method based on k-Means and Hierarchical Clustering, with and without dimensionality reduction. On the other hand, a more exploratory and graph-based method, which included the use of the TensorFlow Embedding Projector (Wongsuphasawat et al., 2018), a Web application that allows for visualizations and analyses of high-dimensional data via Principal Component Analysis (PCA; Shlens, 2014), tdistributed Stochastic Neighbor Embedding (t-SNE; van der Maaten \& Hinton, 2008), and Uniform Manifold Approximation and Projection (UMAP; McInnes et al., 2018). number of clusters from the k-means and hierarchical clustering analyses. The $\mathrm{CH}$ index (Calinski \& Harabasz, 1974) is less sensitive to monotonicity, different cluster densities, subclusters, and skewed distributions. The Silhouette score (Rousseeuw, 1987) is instead more 
234 robust when it comes to handling noisy data, but has difficulty with the presence of subclusters

235 (Liu et al., 2010). The Manhattan distance was used for hierarchical clustering due to the high

236 dimensionality of the dataset (Aggarwal et al., 2001), and three different distance methods were

237 evaluated (simple, complete, and weighted) (SciPy, 2019). Figure S3 shows the obtained CH(k)

238 and sscore $(\mathrm{k})$ for $\mathrm{k}$-means before dimensionality reduction, and indicates that the estimated

239 number of clusters was 2 for both the $\mathrm{CH}$ index and Silhouette score techniques. For hierarchical

240 clustering, the $\mathrm{CH}$ index demonstrated that the best value was 2 for the single and complete

241 distance methods, but 6 for the weighted method, whereas the Silhouette score consistently

242 demonstrated that the best value was 2 (see Figure S3). For both k-means and hierarchical

243 clustering we selected the number of clusters that maximized the score.

245 Once the clustering without dimensionality reduction was done, the dataset was inspected in

246 search of weak and redundant features to mitigate the curse of dimensionality (Jain et al., 2000).

247 To that end, three feature reduction techniques were assessed. First, the PCA revealed that the use of the three strongest singular values would only have explained a modest amount (41\%) of the total variance expressed in the data. Second, the standard deviation plot showed that neither were there fields with zero variation, nor was an exaggerated drop of the variance present in the dataset. Third, the correlation matrix demonstrated that there were some highly correlated diminished by dropping those fields that had a correlation value greater than 0.9 , decreasing the number of dimensions from 207 to $161(22 \%)$. The use of this correlation threshold has been applied in many studies (Katz, 2011). When the CH index and the Silhouette score were used once again to determine the number of clusters, results were unchanged from before 
257 dimensionality reduction (see Figure S4). Finally, k-means and hierarchical clustering were

258 applied according to the obtained number of clusters. Additionally, to facilitate the interpretation

259 of the clustering results, the problem was addressed in a supervised manner, where the

260 membership of the instances to the clusters corresponded to the target classes. To that end, a

261 simple decision tree was trained, and the first decision nodes were analyzed.

262

263

Exploratory approach. The exploratory approach consisted of preparing the input data and

264

265

266

267

268

269

270

271

272

273

274

275

276

277

278

279

\section{2}

exploring the dataset. This meant converting the non-reduced multimodal dataset into a TSV file and creating a metadata file, which enclosed information such as portrayed emotion, valence (positive or negative), actor's ID, and actor's sex. Once both files were loaded into the TensorFlow web application (Wongsuphasawat et al., 2018), data was ready for exploration. The system offers three different primary methods of dimensionality reduction (PCA, t-SNE, and UMAP) and can create two- and three-dimensional plots. For each of these techniques, parameters were tuned until meaningful patterns were found by ocular inspection - zooming in and out on the projections and coloring data points according to metadata.

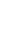

\section{Results}

\section{Supervised learning}

Unimodality. Table 1 lists the best audio classifiers after hyperparameter tuning. Elastic Net with the eGeMAPS parameter set outperformed the rest of the models with an average validation AUC of 0.8196. Most of the classifiers did not suffer from overfitting since their training AUCs were generally close to their correspondent validation ones. However, this was not always the case. Random Forest was prone to reach very high training AUCs and lower validation ones. 
280 This might be because the dataset was too small for an ensemble learning method. Regarding

281 which audio parameter set performed best, there was no clear indication since none of them

282 consistently presented better results. Therefore, both parameter sets were considered during the

283 early fusion approach. Figure S5 presents how the best unimodal audio classifier coped with the

284 validation set in the form of a confusion matrix. The model performed better than chance for all

285 emotions except irritation (chance level performance in an 18-alternative classification task is

$2861 / 18=0.056)$. Additionally, the matrix revealed expected confusion patterns such as confusions

287 between joy and amusement, and between shame and sadness.

Table 2 details the best video classifiers after hyperparameter tuning. Even though Random

290 Forest did a better job than the rest of the classifiers, reaching an average validation AUC of

2910.7981 , it tended to overfit once again. Regarding its intraclass performance, the classifier

292 struggled to recognize some of the emotions, especially shame, which was most of the times

293 mislabeled as sadness. On the other hand, the model stood out in the prediction of amusement

294 samples. The full confusion matrix is available in Figure S6.

295

296

297

298

299

300

301

302

\section{Multimodality}

Late fusion. Once the best audio and video unimodal classifiers were identified, their outputs were merged by using different fusion techniques. Table 3 reveals that product rule outperformed the rest of the methods by achieving an average validation AUC of 0.8767 . The confusion matrix of the best late fusion pipeline (Figure 1) shows that the multimodal classifier performed better than chance for all emotion classes, achieving its highest performance for amusement. Furthermore, it also reveals expected confusion patterns such as confusion between 
303 panic fear and anger, and between contempt and sadness - emotions that belong to the same

304 valence family.

305

306

Early fusion. In this approach, two different input parameter sets were evaluated: an

extended set including the eGeMAPS features and AU intensity values and functionals, and a

minimal set including the standard GeMAPS features and AU intensity and functionals. These

parameter sets were created by concatenating audio and video features and were then used to

train distinct classifiers. Table 4 lists the best early fusion multimodal classifiers after

hyperparameter tuning. Elastic Net with the extended parameter set performed best, scoring an average validation AUC of 0.8662. As shown in Figure 2, its intraclass performance was once again better than random guessing for all emotions, and there were also expected confusion patterns such as confusions between joy and amusement and between anger and panic fear.

\section{Analyses of feature importance}

An in-depth analysis of classifier behavior was conducted by plotting and analyzing the feature importance for each emotion and classifier. Since the input variables were scaled before fitting the model, logistic regression coefficients can be used as feature importance scores for Elastic

Nets. A feature is affecting the prediction when its coefficient is significantly different from zero.

The probability of an event (emotion) increases and decreases when the coefficient is greater or lower than zero, respectively. The behavior of models based on Random Forest was analyzed using the TreeInterpreter package (Saabas, 2015), which decomposes each prediction into bias and feature contribution components. These contributions were grouped by the predicted emotion and then averaged. The interpretation of feature contribution values coincides with the 
326 one explained for Elastic Nets. The contributions of the most important audio features are

327 summarized in Figure 3, and the full list of importance scores for all audio features and emotions

328 is available in Figure S7. A summary of the most important video features (Facial Action Units,

329 AUs) is shown in Figure 4 (with full list shown in Figure S8). These figures are based on the best

330 performing audio and video classifiers and give a detailed look into which features were

331 important for classification of which emotions. They also represent the best performing

332 multimodal classifier, which was based on late fusion of the best unimodal classifiers using the

333 product rule technique.

334

335

In general, different emotions were associated with different patterns of important features.

Important audio features for anger, for example, included spectral balance and amplitude related

features that are associated with a "harsh" voice quality (e.g., spectral slope from 0-500 Hz,

Hammarberg index, harmonics to noise ratio). Whereas for fear, important audio features

included the length of unvoiced segments, amplitude of the first formant frequency, and the

mean slope of falling amplitude signal parts. We refer to Eyben et al. (2016) for definitions and

calculations of audio features. Important video features included AU12 (lip corner puller) for

(upper lip raiser) for amusement. For the sake of completeness, the supplementary materials also include a summary (Figure S9) and full description (Figure S10) of the importance of features for the best early fusion multimodal classification model.

\section{Unsupervised learning}

348 Traditional approach. After determining the optimal number of clusters, these parameters were 
350 over the multimodal dataset, with and without dimensionality reduction. Results from the

351 clustering analyses yielded a two-dimensional solution and are shown in Figure 5.

352

353 In order to interpret the clusters in terms of underlying features, the problem was analyzed in a

354 supervised manner using Decision Trees. As shown in Figure 6, the most relevant feature that

355 distinguished between clusters was AU6 (cheek raiser). According to the first decision node,

356 those instances which had an 80th percentile value greater than 0.328 were classified as cluster

357 one. In the next decision node, AU12 (lip corner puller) also contributed, whereas vocal features

358 became more prominent in the third node. These findings were in good agreement with the

359 emotion categories included in cluster one (see Figure 5), which mainly comprised emotions that

360 are positive (and are characterized by the use of AU6, e.g., amusement and joy; see Figure S8).

361 The pattern was consistent across both clustering techniques without dimensionality reduction,

362 but changed when the reduced dataset was used for hierarchical clustering (see Figure 5, bottom

363 right).

364

365 Exploratory approach. After preparing the features and metadata files of the non-reduced

366

367

368

369

370

371 multimodal dataset (as described in the Methods section), the data was explored in search of

meaningful patterns. To this end, three different dimensionality reduction techniques were employed via the TensorFlow Embedding Projector (Wongsuphasawat et al., 2018). The tunable

parameters were manually adjusted until any interesting patterns were found. The reader can interactively visualize the data and inspect the results ${ }^{1}$. Main findings are presented below.

${ }^{1}$ https://projector.tensorflow.org/?config=https://raw.githubusercontent.com/marferca/multimodal-emotionrecognition/main/4.unsupervised learning/exploratory approach/tf embedding projector/projector config.json 
space reduced the amount of explained variance to $35 \%$, some interesting patterns were detected.

374 It is apparent from Figure 7 that the dataset could be split into two clusters. The left and middle

375 side mainly contained high-arousal emotions, such as portrayals of amusement, anger, joy, panic

376 fear, pride, and despair. The right side instead included low-arousal emotions, such as sadness,

377 irritation, interest, anxiety, and contempt. In addition, representations of the same emotion

378 tended to be close to each other.

$\boldsymbol{t}-\boldsymbol{S N E}$. The t-SNE dimensionality reduction technique was run until convergence $(6,081$ colored by emotion, valence, actor, and actor's sex. The algorithm grouped the data into three main clusters (Figure 8), in which emotions characterized by positive valence were grouped together, whereas the rest split into two clusters with high prevalence of panic fear and anger, and of sadness, respectively. Figure 8 also reveals that emotions portrayed by the same actor tended to be close to each other. Something similar also happened when coloring by sex: female samples tended to group together, as did male samples.

$\boldsymbol{U M A P}$. The UMAP algorithm was run for 500 epochs (not a tunable parameter) and 36 neighbors. As shown in Figure 9, the left side mainly contained high-arousal emotions and positive emotions (e.g., amusement, joy, and despair), whereas the right side contained lowarousal emotions and negative emotions (e.g., sadness, irritation, anxiety, contempt, and interest). Similarly to t-SNE, expressions portrayed by the same actor were close to each other

394 (e.g., actor number 3, pink dots, is a case in point). Finally, Figure 9 also reveals how the actor's 
395

396

397

398

399

400

401

402

403

404

405

406

407

408

409

410

411

412

413

414

415

416

417

418

sex played a part in the clustering results since most of the female and male samples were on the upper and the lower part of the output, respectively.

\section{Discussion}

We investigated classification of 18 emotions - portrayed by 10 actors through vocal and facial expressions - using person-independent supervised and unsupervised methods. Our study makes three main contributions to the literature. First, results from the supervised experiments showed that multimodal classifiers performed better than unimodal classifiers and were able to classify all emotions, although recognition rates varied widely across emotions. This indicates that the combinations of vocal and facial features that were used for classification varied systematically as a function of emotion, and that the signal was reliable enough to allow for above chance classification of all 18 emotions. Second, we utilized our machine learning approach to present new data on multimodal feature patterns for each emotion, in terms of the features that contributed most to classifier performance. Third, we explored how wholly unsupervised classifiers would organize the emotion expressions, based on the same features that were used for supervised classification. Several meaningful explanatory patterns were observed and interpreted in terms of valence, arousal, and various actor- and sex-specific aspects. The comparison of supervised and unsupervised approaches allowed us to explore how different methodological choices may provide different perspectives on how emotion expressions are organized.

Overall, the multimodal classifiers performed approximately 5-6\% better than the unimodal vocal and facial classifiers in our supervised experiments. The magnitude of this improvement is in accordance with previous studies (see D'Mello \& Kory, 2015, for a review). We observed the 
419 best performance $(\mathrm{AUC}=0.88)$ for multimodal classifiers that merged the output from the best

420 unimodal vocal (elastic net, $\mathrm{AUC}=0.82$ ) and facial (random forest, $\mathrm{AUC}=0.80$ ) classifiers

421 using a late fusion approach and the product rule method. A direct comparison with previous

422 classification studies on the GEMEP expression set is difficult because studies have used

423 different classification approaches (e.g., person-independent or person-dependent), algorithms,

424 numbers of emotion categories, and selections of stimuli. Our unimodal vocal and facial

425 classifiers seemed to achieve slightly lower accuracy compared to previous efforts (e.g., Schuller

426 et al., 2019; Valstar et al., 2012), although it must be noted that earlier studies have classified

427 fewer emotion categories. We also used relatively small feature sets, with the aim of mainly

428 including features that are possible to interpret in terms of human perception. For example, we

429 focused on AUs because they provide a comprehensive and widely used way to describe facial

430 expressions (Martinez et al., 2019), that can be used to produce easily interpretable feature

431 patterns for each emotion. However, inclusion of additional features such as head pose and gaze

432 direction would likely increase classification performance. Recent studies have also abandoned

433 the use of pre-defined features altogether and instead use deep learning of physical properties

434 with good results (e.g., Li \& Deng, 2020), although such methods often result in features that are 435 difficult to interpret.

437 Bänziger and Scherer (2010) provided data on human classification for the same stimulus set as

438 used in the current study. Direct comparison of recognition rates is again difficult because the

439 human judgments were collected using different methodology (e.g., judges were allowed to

440 choose more than one alternative in a forced-choice task), but overall our classifiers had

441 somewhat lower recognition rates than the human judges. However, an inspection of recognition 
442 patterns showed many similarities between human judges and classifiers. For example, the

443 human judges in Bänziger and Scherer (2010), like our classifiers, received higher accuracy for

444 multimodal vs unimodal expressions. Looking at individual emotions, human judges showed

445 highest accuracy for amusement, anger, and panic fear. Our classifiers also performed best for

446 amusement and anger, and also performed relatively well for panic fear. Shame received the

447 lowest recognition rates from both human judges and our classifiers. Even confusion patterns

448 showed many similarities between human judges and classifiers. For example, joy and

449 amusement, tenderness and pleasure, relief and pleasure, and despair and panic fear were among

450 the most frequent confusions for both humans and classifiers. Similar recognition patterns for

451 human judges and classifiers tentatively suggest that the included features may also be relevant

452 for human perception of emotion.

453

454 Traditional emotion recognition studies using machine learning often aim to achieve the highest 455 possible classification performance, and do not pay attention to feature importance. We propose 456 that a more detailed inspection of feature importance presents a promising method for

457 investigating emotion-related patterns of probabilistic and partly redundant vocal and facial

458 features. Such patterns may be difficult to discover using traditional descriptive statistics and

459 linear analyses. We present detailed multimodal feature patterns for each of the 18 included 460 emotions, several of which have rarely been included in previous emotion expression studies

461 (e.g., Figure 3 and Figure 4). Future studies are needed to investigate how well the obtained 462 patterns may generalize to other data sets and other classification methods. 
464 We also performed a number of unsupervised classification experiments, guided by the idea that

465 they may provide additional information about how emotion expressions are organized (e.g.,

466 Azari et al., 2020). Previous studies on the organization of emotion expressions have focused on

467 human perception (e.g., Cowen et al., 2019), whereas our study instead investigated the

468 organization of emotion expressions based on their physical vocal and facial properties. Results

469 from these experiments did not replicate a structure with 18 emotion categories. This was

470 expected because such a solution would require that almost all of the variance in the included

471 features would be explained by emotion expressions. However, all methods lead to meaningful

472 structures that could often be interpreted in relation to emotion categories. Traditional methods

473 based on k-means and hierarchical clustering proposed a two-factor solution which was

474 interpreted using decision trees. This approach revealed that AU6 (cheek raiser) was the most

475 relevant feature at the first decision node. This guided our interpretation of the two clusters as

476 largely representing positive and negative valence, although expressions of negative emotions

477 that shared key features with positive emotions did also end up in the 'positive' cluster. The

478 exploratory dimension reduction methods gave further insights. PCA results could largely be

479 interpreted in terms of high vs. low arousal expressions, although Figure 7 also revealed that

480 portrayals of the same emotion tended to be close together. Results from the t-SNE and UMAP

481 analyses could also be interpreted in terms of arousal, valence, and emotion - but they also

482 revealed that person and gender specific aspects contributed to clustering. For example,

483 portrayals from the same actor tended to be close to each other, as did portrayals by male and

484 female actors, respectively (see Figure 8 and Figure 9). One conclusion that can be drawn is that

485 both emotional and non-emotional variability is likely to play a role in unsupervised

486 classification of emotions (see Li \& Deng, 2020), especially in person-independent approaches 
487 where within-person normalization of features is often not a feasible solution. Future research

488 could focus on ways to minimize the impact of feature variability that is not directly related to

489 the expression of emotions.

490

491 Our stimuli consisted of actor portrayals recorded in a studio, so future research is required to

492 investigate which of the results will generalize to naturalistic conditions. Studies using

493 spontaneous expressions are important because recent research suggests that there may be small

494 but systematic differences between how emotions are conveyed in actor portrayals vs.

495 spontaneous expressions (e.g., Juslin et al., 2018; Krumhuber et al., 2021a). Another limitation

496 of our study was that we did not fully take advantage of the dynamic nature of the stimuli, and

497 only used such temporal dynamics information that was directly encoded in the features. Future

498 studies could instead track the dynamic changes of features over time and use analysis methods

499 that take advantage of this information (e.g., long short-term memory recurrent neural networks;

500 see Wöllmer et al., 2013; Zhao et al., 2019). While the GEMEP data set is relatively small,

501 openly available huge data sets could in the future enable modeling of emotion expressions via

502 transformers and attention-based mechanisms (e.g., Siriwardhana et al., 2020; Vaswani et al.,

503 2017). Even for moderately sized data sets, such modeling could be useful, as we have recently

504 shown in another context (Gogoulou et al., 2021).

505

506 With an increase in the number of human-robot interactions with an emotional component

507 (Shum et al., 2018) - e.g., recognizing an angry customer in dialog with a chatbot - the future

508 will hold more opportunities for data-driven reasoning. Besides software robots, there is also an

509 increase in the number of filmed interactions between humans and physical robots and

PeerJ Comput. Sci. reviewing PDF | (CS-2021:06:62753:1:1:NEW 3 Sep 2021) 
510 corresponding studies of human-robot cooperation (Crandall et al., 2018). Studies into unlabeled

511 data from such sources can be done using self-supervised learning. Such studies may assist in

512 understanding the importance of correctly interpreting emotions, and will likely also become

513 more common and potentially have important societal implications. This further motivates deep

514 dives into the methodology, of the kind that we have attempted here.

515

516 To conclude, we propose that research on emotion expression could benefit from augmenting

517 supervised methods with unsupervised clustering. The combination of methods leads to more

518 insight into how data is organized, and we especially note that the investigation of explanatory

519 patterns could be a valuable meta-heuristic that can be applied to several classification areas. We

520 believe that advances in the basic science of understanding how emotions are expressed requires

521 continued efforts regarding both the development of more representative stimulus materials and

522 of more representative vocal and facial features. Machine learning methods could play a vital

523 role in the study of how emotions are expressed nonverbally through the voice and the face.

524

525

526

527

528

529

530

531

532

Peer] Comput. Sci. reviewing PDF | (CS-2021:06:62753:1:1:NEW 3 Sep 2021) 
534

535

536

537

538

539

540

\section{References}

541

542

543

Aggarwal, C. C., Hinneburg, A., \& Keim, D. A. (2001). On the surprising behavior of distance metrics in high dimensional space. In J. Van den Bussche \& V. Vianu (Eds.), Database theoryICDT 2001 (pp. 420-434). Springer. 10.1007/3-540-44503-X_27

Atrey, P. K., Hossain, M. A., El Saddik, A., \& Kankanhalli, M. S. (2010). Multimodal fusion for multimedia analysis: A survey. Multimedia Systems, 16(6), 345-379. 10.1007/s00530-010-0182$\underline{0}$

549 Comparing supervised and unsupervised approaches to emotion categorization in the human brain, body, and subjective experience. Scientific Reports, 10, Article 20284. 10.1038/s41598$\underline{020-77117-8}$

557

Baltrušaitis, T, Mahmoud, M., \& Robinson, P. (2015). Cross-dataset learning and person-specific normalisation for automatic Action Unit detection. In 11th IEEE International Conference and Workshops on Automatic Face and Gesture Recognition (FG 2015) (pp. 1-6). IEEE. doi: $\underline{10.1109 / F G .2015 .7284869}$

Baltrušaitis, T., Zadeh, A., Lim, Y. C., \& Morency, L. P. (2018). Openface 2.0: Facial behavior Recognition (FG 2018) (pp. 59-66). IEEE. 10.1109/FG.2018.00019 
569 Bänziger, T., \& Scherer, K. R. (2010). Introducing the Geneva Multimodal Emotion Portrayal 570 (GEMEP) corpus. In K. R. Scherer, T. Bänziger, \& E. B. Roesch (Eds.), Blueprint for affective 571 computing: A sourcebook (pp. 271-294.). Oxford University Press.

572

573

Barrett, L. F., Adolphs, R., Marsella, S., Martinez, A. M., \& Pollak, S. D. (2019). Emotional expressions reconsidered: Challenges to inferring emotion from human facial movements.

575

576

577

578 Psychological Science in the Public Interest, 20(1), 1-68. 10.1177/1529100619832930

Bhattacharya, P., Gupta, R. K., \& Yang, Y. (2021). Exploring the contextual factors affecting multimodal emotion recognition in videos. IEEE Transactions on Affective Computing. $\underline{10.1109 / \text { TAFFC.2021.3071503 }}$

580

Calinski, T., \& Harabasz, J. (1974). A dendrite method for cluster analysis. Communications in

Cordaro, D. T., Sun, R., Keltner, D., Kamble, S., Huddar, N., \& McNeil, G. (2018). Universals and cultural variations in 22 emotional expressions across five cultures. Emotion, 18(1), 75-93. $\underline{10.1037 / \mathrm{emo} 0000302}$

Cowen, A. S., Laukka, P., Elfenbein, H. A., Liu, R., \& Keltner, D. (2019). The primacy of categories in the recognition of 12 emotions in speech prosody across two cultures. Nature Human Behaviour, 3(4), 369-382. 10.1038/s41562-019-0533-6

Crandall, J.W., Oudah, M., Tennom, Ishowo-Oloko, F., Abdallah, S., Bonnefon, J-F., Cebrian, M., Shariff, A., Goodrich, M. A., \& Rahwan, I. (2018). Cooperating with machines. Nature Communications, 9, Article 233. 10.1038/s41467-017-02597-8

595

596

597

598

D’Mello, S. K., \& Kory, J. (2015). A review and meta-analysis of multimodal affect detection systems. ACM Computing Surveys, 47(3), 43-79. 10.1145/2682899

Dong, X. L., Gabrilovich, E., Heitz, G., Horn, W., Murphy, K., Sun, S., \& Zhang, W. (2015).

600

601 From data fusion to knowledge fusion. Proceedings of the VLDB Endowment, 7(10), 881-892. $\underline{10.14778 / 2732951.2732962}$

602

603

604

605

606

607

608

609

610

Ekman, P. (2003). Emotions revealed. Henry Holt.

Ekman, P., \& Friesen, W. V. (1978). Facial action coding system: A technique for the measurement of facial movement. Consulting Psychologists Press.

Elfenbein, H. A., \& Ambady, N. (2002). On the universality and cultural specificity of emotion recognition: A meta-analysis. Psychological Bulletin, 128(2), 203-235. 10.1037/00332909.128.2.203

611 Eyben, F., Scherer, K. R., Schuller, B. W., Sundberg, J., André, E., Busso, C., Devillers, L. Y., 612 Epps, J., Laukka, P., Narayanan, S. S., \& Truong, K. P. (2016). The Geneva Minimalistic 
613 Acoustic Parameter Set (GeMAPS) for voice research and affective computing. IEEE

614 Transactions on Affective Computing, 7(2), 190-202. 10.1109/TAFFC.2015.2457417

615 Eyben, F., Weninger, F., Gross, F., \& Schuller, B. (2013). Recent developments in openSMILE, 616 the Munich open-source multimedia feature extractor. In A. Jaimes, N. Sebe, N. Boujemaa, D.

617 Gatica-Perez, D. A. Shamma, M. Worring, \& R. Zimmermann (Eds.), Proceedings of the 21st

618 ACM International Conference on Multimedia (pp. 835-838). ACM. 10.1145/2502081.2502224

619

620 Gogoulou, E., Boman, M., Abdesslem, F. B., Isacsson, N. H., Kaldo, V., \& Sahlgren, M. (2021).

621 Predicting treatment outcome from patient texts: The case of internet-based cognitive

622 behavioural therapy. In Proceedings of the 16th Conference of the European Chapter of the

623 Association for Computational Linguistics: Main Volume (pp. 575-580). Association for

624 Computational Linguistics. https://aclanthology.org/2021.eacl-main.46

625

626

627

628

629

630

Hofmann, S. G. (2016). Emotion in therapy: From science to practice. Guilford Press.

Jain, A. K., Duin, R. P. W., \& Mao, J. (2000). Statistical pattern recognition: A review. IEEE

Transactions on Pattern Analysis and Machine Intelligence, 22(1), 4-37. 10.1109/34.824819

Jeni, L. A., Cohn, J. F., \& De La Torre, F. (2013). Facing imbalanced data recommendations for the use of performance metrics. In Proceedings of the 2013 Humaine Association Conference on Affective Computing and Intelligent Interaction (ACII 2013) (pp. 245-251). IEEE. $\underline{10.1109 / A C I I .2013 .47}$

Jeon, M. (2017). Emotions and affect in human factors and human-computer interaction. Academic Press.

Juslin, P. N., \& Laukka, P. (2003). Communication of emotion in vocal expression and music performance: Different channels, same code? Psychological Bulletin, 129(5), 770-814. $\underline{10.1037 / 0033-2909.129 .5 .770}$

Juslin, P. N., Laukka, P., \& Bänziger, T. (2018). The mirror to our soul? Comparisons of spontaneous and posed vocal expression of emotion. Journal of Nonverbal Behavior, 42(1), 140. 10.1007/s10919-017-0268-X

Katz, M. (2011). Multivariable analysis: A practical guide for clinicians and public health researchers. Cambridge University Press.

Krumhuber, E. G., Küster, D., Namba, S., Shah, D., \& Calvo, M. G. (2021a). Emotion recognition from posed and spontaneous dynamic expressions: Human observers versus machine analysis. Emotion, 21(2), 447-451. 10.1037/emo0000712

655

Krumhuber, E.G., Küster, D., Namba, S., \& Skori, L. (2021b). Human and machine validation of 656 14 databases of dynamic facial expressions. Behavior Research Methods, 53, 686-701.

657 $\underline{10.3758 / \mathrm{s} 13428-020-01443-\mathrm{y}}$ 
658 Laukka, P., \& Elfenbein, H. A. (2021). Cross-cultural emotion recognition and in-group

659 advantage in vocal expression: A meta-analysis. Emotion Review, 13(1), 3-11. doi:

$660 \quad \underline{10.1177 / 1754073919897295}$

661

662

663

664

665

666

667

668

669

670

671

672

673

674

675

676

677

678

679

680

681

682

683

684

685

686

687

688

689

690

691

692

693

694

695

696

697

698

699

700

701

702

703
Li, S., \& Deng, W. (2020). Deep facial expression recognition: A survey. IEEE Transactions on Affective Computing. Early access. 10.1109/TAFFC.2020.2981446

Lingenfelser, F., Wagner, J., Deng, J., Brueckner, R., Schuller, B., \& André, E. (2018).

Asynchronous and event-based fusion systems for affect recognition on naturalistic data in comparison to conventional approaches. IEEE Transactions on Affective Computing, 9, 410-423. $\underline{10.1109 / T A F F C .2016 .2635124 ~}$

Liu, Y., Li, Z., Xiong, H., Gao, X., \& Wu, J. (2010). Understanding of internal clustering validation measures. In 2010 IEEE International Conference on Data Mining (pp. 911-916). IEEE. 10.1109/ICDM.2010.35

Mai, S., Hu, H., Xu, J., \& Xing, S. (2020). Multi-fusion residual memory network for multimodal human sentiment comprehension. IEEE Transactions on Affective Computing. Early access. 10.1109/TAFFC.2020.3000510

Marechal, C., Mikołajewski, D., Tyburek, K., Prokopowicz, P., Bougueroua, L., Ancourt, C., \& Wegrzyn-Wolska, K. (2019). Survey on AI based multimodal methods for emotion detection. In J. Kołodziej \& H. González-Vélez (Eds.) High-performance modelling and simulation for big data applications (pp. 307-324). Springer. 10.1007/978-3-030-16272-6 11

Martinez, B., Valstar, M. F., Jiang, B., \& Pantic, M. (2019). Automatic analysis of facial actions: A survey. IEEE Transactions on Affective Computing, 10(3), 325-347. $\underline{10.1109 / T A F F C .2017 .2731763}$

McInnes, L., Healy, J., \& Melville, J. (2018). UMAP: Uniform manifold approximation and projection for dimension reduction. arXiv. https://arxiv.org/abs/1802.03426

Poria, S., Cambria, E., Bajpai, R., \& Hussain, A. (2017). A review of affective computing: From unimodal analysis to multimodal fusion. Information Fusion, 37, 98-125.

$\underline{10.1016 / \text { j.inffus.2017.02.003 }}$

Rousseeuw, P. J. (1987). Silhouettes: A graphical aid to the interpretation and validation of cluster analysis. Journal of Computational and Applied Mathematics, 20, 53-65. 10.1016/0377$\underline{0427(87) 90125-7}$

Russell, J. A., Bachorowski, J. A., \& Fernandez-Dols, J. M. (2003). Facial and vocal expressions of emotion. Annual Review of Psychology, 54, 329-349.

10.1146/annurev.psych.54.101601.145102

Saabas, A. (2015). TreeInterpreter [Computer software]. https:/github.com/andosa/treeinterpreter

Peer] Comput. Sci. reviewing PDF | (CS-2021:06:62753:1:1:NEW 3 Sep 2021) 
704

705

706

707

708

709

710

711

712

713

714

715

716

717

718

719

720

721

722

723

724

725

726

727

728

729

730

731

732

733

734

735

736

737

738

739

740

741

742

743

744

745

746

747

748

749
Scherer, K. R. (2009) Emotion theories and concepts (psychological perspectives). In D. Sander \& K R. Scherer (Eds.), Oxford companion to emotion and the affective sciences (pp. 145-151). Oxford University Press.

Schuller, B. (2018). Speech emotion recognition: Two decades in a nutshell, benchmarks, and ongoing trends. Communications of the ACM, 61(5), 90-99. 10.1145/3129340

Schuller, B., Weninger, F., Zhang, Y., Ringeval, F., Batliner, A., Steidl, S., Eyben, F., Marchi, E., Vinciarelli, A., Scherer, K., Chetouani, M., \& Mortillaro, M. (2019). Affective and behavioural computing: Lessons learnt from the First Computational Paralinguistics Challenge. Computer Speech and Language, 53, 156-180. 10.1016/j.csl.2018.02.004

SciPy (2019). Hierarchical clustering (scipy.cluster.hierarchy.linkage).

https://docs.scipy.org/doc/scipy/reference/generated/scipy.cluster.hierarchy.linkage.html

[Accessed: 28 June 2020].

Shlens, J. (2014). A tutorial on principal component analysis. arXiv.

https://arxiv.org/abs/1404.1100

Shum, H-Y., He, X-D., \& Li, D. (2018). From Eliza to XiaoIce: Challenges and opportunities with social chatbots. Frontiers of Information Technology and Electronic Engineering, 19, 10 26. 10.1631/FITEE.1700826

Siriwardhana, S., Kaluarachchi, T., Billinghurst, M., \& Nanayakkara, S. (2020). Multimodal emotion recognition with transformer-based self supervised feature fusion. IEEE Access, 8 , 176274-176285. 10.1109/ACCESS.2020.3026823

Sjardin, B., Massaron, L., \& Boschetti, A. (2016). Large scale machine learning with Python. Packt Publishing.

Srinivasan, R., \& Martinez, A. M. (2018). Cross-cultural and cultural-specific production and perception of facial expressions of emotion in the wild. IEEE Transactions on Affective Computing. Early access. 10.1109/TAFFC.2018.2887267

Tzirakis, P., Trigeorgis, G., Nicolaou, M. A., Schuller, B. W., \& Zafeiriou, S. (2017). End-to-end multimodal emotion recognition using deep neural networks. IEEE Journal of Selected Topics in Signal Processing, 11(8), 1301-1309. 10.1109/JSTSP.2017.2764438

Valstar, M. F., Mehu, M., Jiang, B., Pantic, M., \& Scherer, K. (2012). Meta-analysis of the First Facial Expression Recognition Challenge. IEEE Transactions on Systems, Man, and Cybernetics, Part B (Cybernetics), 42(4), 966-979. 10.1109/TSMCB.2012.2200675

van der Maaten, L., \& Hinton, G. (2008). Visualizing data using t-sne. Journal of Machine Learning Research, 9, 2579-2605.

https://www.jmlr.org/papers/volume9/vandermaaten08a/vandermaaten08a.pdf

Peer] Comput. Sci. reviewing PDF | (CS-2021:06:62753:1:1:NEW 3 Sep 2021) 
750

751

752

753

754

755

756

757

758

759

760

761

762

763

764

765

766

767

768

769

770

771

772

773

774

775

776

777

778

779

780
Vaswani, A., Shazeer, N., Parmar, N., Uszkoreit, J., Jones, L., Gomez, A. N., Kaiser, Ł., \& Polosukhin, I. (2017). Attention is all you need. In I. Guyon, U. V. Luxburg, S. Bengio, H. Wallach, R. Fergus, S. Vishwanathan, \& R. Garnett (Eds.), Advances in neural information processing systems 30 (NIPS 2017) (pp. 5998-6008). Neural Information Processing Systems Foundation. https://papers.nips.cc/paper/7181-attention-is-all-you-need

Wang, Z., Wang, L., \& Huang, H. (2020). Joint low rank embedded multiple features learning for audio-visual emotion recognition. Neurocomputing, 388, 324-333.

$\underline{10.1016 / \text { j.neucom.2020.01.017 }}$

Wöllmer, M., Kaiser, M., Eyben, F., Schuller, B., \& Rigoll, G. (2013). LSTM-modeling of continuous emotions in an audiovisual affect recognition framework. Image and Vision Computing, 31(2), 153-163. 10.1016/j.imavis.2012.03.001

Wongsuphasawat, K., Smilkov, D., Wexler, J., Wilson, J., Mané, D., Fritz, D., Krishnan, D., Viégas, F. B., \& Wattenberg, M. (2018). Visualizing dataflow graphs of deep learning models in TensorFlow. IEEE Transactions on Visualization and Computer Graphics, 24(1), 1-12. $\underline{10.1109 / T V C G .2017 .2744878}$

Zhao, Z., Bao, Z., Zhao, Y., Zhang, Z., Cummins, N., Ren, Z., \& Schuller, B. (2019). Exploring deep spectrum representations via attention-based recurrent and convolutional neural networks for speech emotion recognition. IEEE Access, 7, 97515-97525. 10.1109/ACCESS.2019.2928625 


\section{Table $\mathbf{1}$ (on next page)}

Summary of unimodal audio classification experiments. 
1

\begin{tabular}{|l|c|c|}
\hline Classifier & AUC (train) & AUC (validation) \\
\hline Elastic Net (eGeMAPS) & $\mathbf{0 . 9 1 3 6} \pm \mathbf{0 . 0 0 1 8}$ & $\mathbf{0 . 8 1 9 6} \pm \mathbf{0 . 0 1 9 2}$ \\
\hline Elastic Net (GeMAPS) & $0.9034 \pm 0.0028$ & $0.8086 \pm 0.0223$ \\
\hline k-NN (eGeMAPS) & $0.8244 \pm 0.0056$ & $0.7764 \pm 0.0199$ \\
\hline k-NN (GeMAPS) & $0.8164 \pm 0.0056$ & $0.7761 \pm 0.0187$ \\
\hline Decision Tree (eGeMAPS) & $0.7742 \pm 0.0068$ & $0.7019 \pm 0.0229$ \\
\hline Decision Tree (GeMAPS) & $0.7695 \pm 0.0073$ & $0.7029 \pm 0.0331$ \\
\hline Random Forest (eGeMAPS) & $0.9983 \pm 0.0003$ & $0.7979 \pm 0.0208$ \\
\hline Random Forest (GeMAPS) & $0.9967 \pm 0.0004$ & $0.7991 \pm 0.0189$ \\
\hline
\end{tabular}

2 


\section{Table 2 (on next page)}

Summary of unimodal video classification experiments. 
1

\begin{tabular}{|l|c|c|}
\hline Classifier & AUC (training) & AUC (validation) \\
\hline Elastic Net & $0.8685 \pm 0.0047$ & $0.7946 \pm 0.0254$ \\
\hline k-NN & $0.8216 \pm 0.0074$ & $0.7780 \pm 0.0309$ \\
\hline Decision Tree & $0.7449 \pm 0.0070$ & $0.6989 \pm 0.0255$ \\
\hline Random Forest & $\mathbf{0 . 9 9 9 2} \pm \mathbf{0 . 0 0 0 1}$ & $\mathbf{0 . 7 9 8 1} \pm \mathbf{0 . 0 2 3 8}$ \\
\hline
\end{tabular}

2

3

4

5

6 


\section{Table 3 (on next page)}

Summary of multimodal classification experiments using the late fusion approach. 
1

\begin{tabular}{|l|c|c|}
\hline Classifier & AUC (training) & AUC (validation) \\
\hline Maximum Rule & $0.9960 \pm 0.0003$ & $0.8360 \pm 0.0116$ \\
\hline Sum Rule & $0.9972 \pm 0.0002$ & $0.8618 \pm 0.0127$ \\
\hline Product Rule & $\mathbf{0 . 9 9 7 2} \pm \mathbf{0 . 0 0 0 2}$ & $\mathbf{0 . 8 7 6 7} \pm \mathbf{0 . 0 1 3 5}$ \\
\hline Weight Criterion & $0.9981 \pm 0.0002$ & $0.8623 \pm 0.0129$ \\
\hline Rule-based & $0.9653 \pm 0.0015$ & $0.8470 \pm 0.0176$ \\
\hline Elastic Net & $0.9994 \pm 0.0000$ & $0.8696 \pm 0.0168$ \\
\hline k-NN & $0.9825 \pm 0.0007$ & $0.8585 \pm 0.0160$ \\
\hline Decision Tree & $0.8042 \pm 0.0096$ & $0.7079 \pm 0.0267$ \\
\hline
\end{tabular}

2

3

4

5

6

7

8

9

10 


\section{Table 4 (on next page)}

Summary of multimodal classification experiments using the early fusion approach.

ext $=$ extended feature set $($ eGeMAPS + AUs $) ; \min =$ minimal feature set $($ GeMAPS + AUs $)$. 
1

\begin{tabular}{|l|c|c|}
\hline Classifier & AUC (train) & AUC (validation) \\
\hline Elastic Net (ext.) & $\mathbf{0 . 9 6 3 4} \pm \mathbf{0 . 0 0 2 4}$ & $\mathbf{0 . 8 6 6 2} \pm \mathbf{0 . 0 1 5 0}$ \\
\hline Elastic Net (min.) & $0.9401 \pm 0.0029$ & $0.8600 \pm 0.0174$ \\
\hline k-NN (ext.) & $0.8764 \pm 0.0057$ & $0.8334 \pm 0.0255$ \\
\hline k-NN (min.) & $0.8476 \pm 0.0077$ & $0.8285 \pm 0.0259$ \\
\hline Decision Tree (ext.) & $0.7772 \pm 0.0093$ & $0.7320 \pm 0.0280$ \\
\hline Decision Tree (min.) & $0.7936 \pm 0.0130$ & $0.7321 \pm 0.0376$ \\
\hline Random Forest (ext.) & $0.9998 \pm 0.0000$ & $0.8550 \pm 0.0222$ \\
\hline Random Forest (min.) & $1.0000 \pm 0.0000$ & $0.8555 \pm 0.0225$ \\
\hline
\end{tabular}

2

3 Note. ext $=$ extended feature set $($ eGeMAPS + AUs $) ; \min =$ minimal feature set $($ GeMAPS + 4 AUs). 


\section{Figure 1}

Confusion matrix showing the proportion of responses in the validation set for the best late fusion multimodal classifier (product rule).

Recall rates are shown in the diagonal cells (marked in bold). $\mathrm{adm}=$ admiration, $\mathrm{amu}=$ amusement, ang = anger, anx $=$ anxiety/worry, con $=$ contempt, des $=$ despair, dis $=$ disgust, fea $=$ panic fear, int $=$ interest, irr $=$ irritation, joy $=$ joy/elation, ple $=$ sensual pleasure, pri $=$ pride, rel $=$ relief, sad $=$ sadness, sha $=$ shame, sur $=$ surprise, ten $=$ tenderness. 


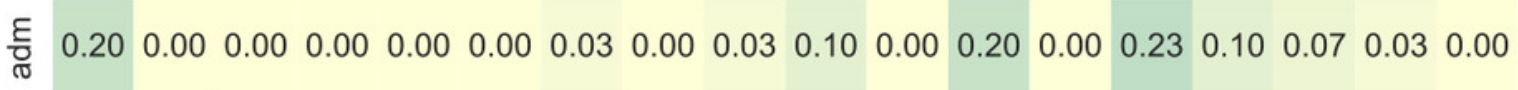
ᄅ⿱

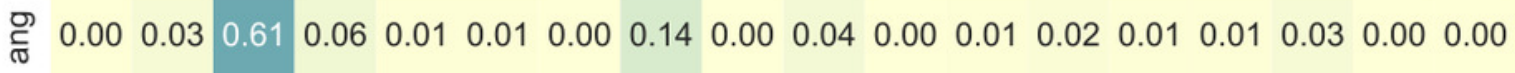

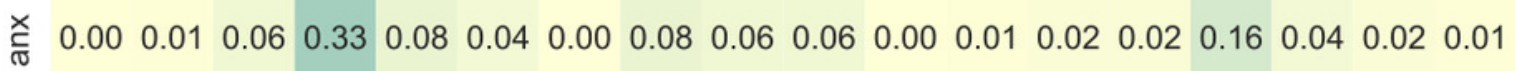
등 $\begin{array}{lllllllllllllllllll}0.00 & 0.00 & 0.00 & 0.10 & 0.30 & 0.00 & 0.00 & 0.00 & 0.00 & 0.17 & 0.00 & 0.03 & 0.00 & 0.00 & 0.27 & 0.07 & 0.07 & 0.00\end{array}$ $\begin{array}{lllllllllllllllllll}\text { \& } & 0.00 & 0.08 & 0.03 & 0.03 & 0.04 & 0.33 & 0.00 & 0.19 & 0.01 & 0.07 & 0.09 & 0.01 & 0.02 & 0.00 & 0.04 & 0.03 & 0.01 & 0.00\end{array}$

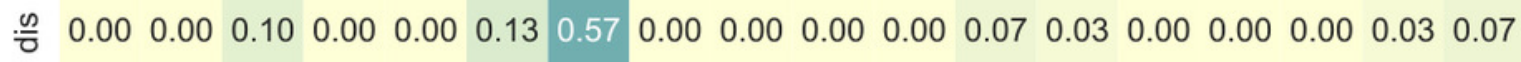

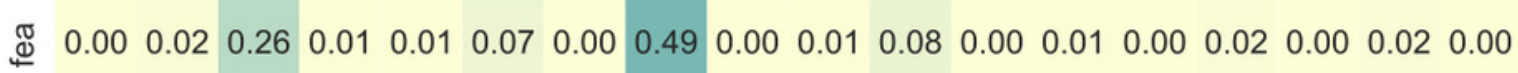

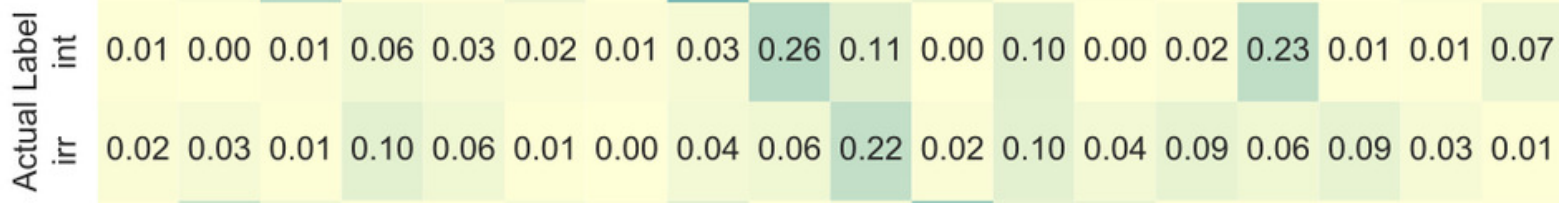
$\begin{array}{lllllllllllllllllll}\text {. } & 0.00 & 0.19 & 0.06 & 0.01 & 0.00 & 0.03 & 0.01 & 0.10 & 0.00 & 0.03 & 0.38 & 0.09 & 0.08 & 0.01 & 0.01 & 0.00 & 0.00 & 0.00\end{array}$

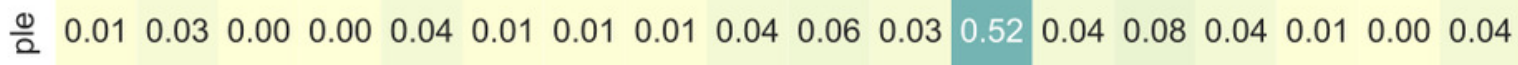

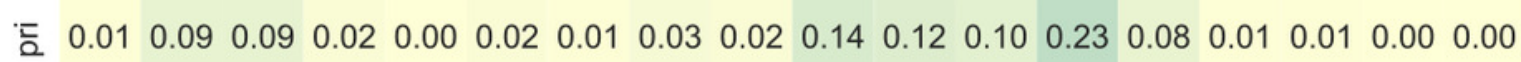
\begin{tabular}{lllllllllllllllllll}
\hline & 0.01 & 0.00 & 0.00 & 0.03 & 0.00 & 0.03 & 0.01 & 0.00 & 0.03 & 0.13 & 0.01 & 0.23 & 0.06 & 0.34 & 0.02 & 0.06 & 0.02 & 0.00
\end{tabular}

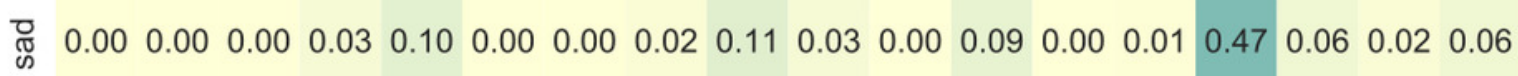

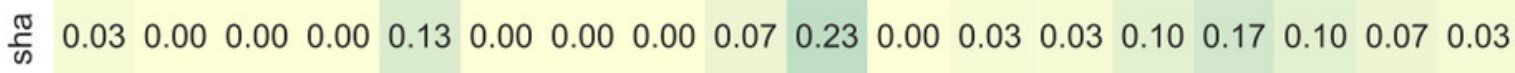

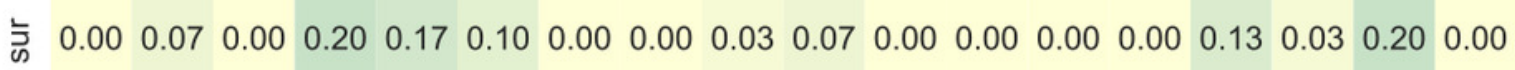

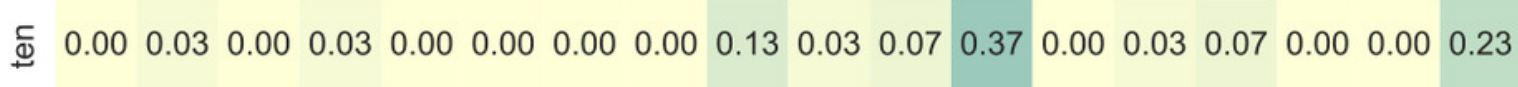
adm amu ang anx con des dis fea int irr joy ple pri rel sad sha sur ten 


\section{Figure 2}

Confusion matrix showing the proportion of responses in the validation set for the best early fusion multimodal classifier (Elastic Net with extended feature set).

Recall rates are shown in the diagonal cells (marked in bold). $\mathrm{adm}=$ admiration, $\mathrm{amu}=$ amusement, ang = anger, anx $=$ anxiety/worry, con $=$ contempt, des $=$ despair, dis $=$ disgust, fea $=$ panic fear, int $=$ interest, irr $=$ irritation, joy $=$ joy/elation, ple $=$ sensual pleasure, pri $=$ pride, rel $=$ relief, sad $=$ sadness, sha $=$ shame, sur $=$ surprise, ten $=$ tenderness. 
छ

$\begin{array}{llllllllllllllllll}0.27 & 0.00 & 0.00 & 0.03 & 0.00 & 0.00 & 0.00 & 0.00 & 0.03 & 0.17 & 0.03 & 0.07 & 0.03 & 0.23 & 0.10 & 0.03 & 0.00 & 0.00\end{array}$

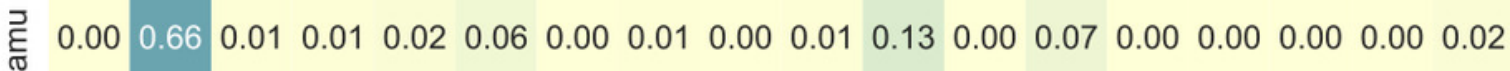

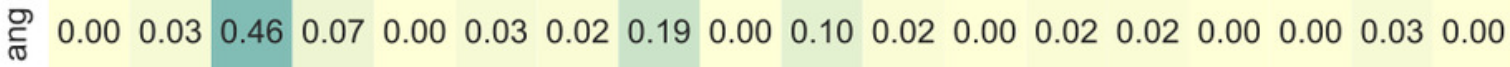

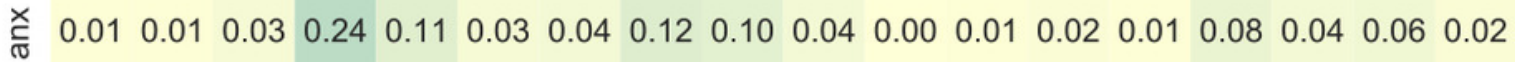

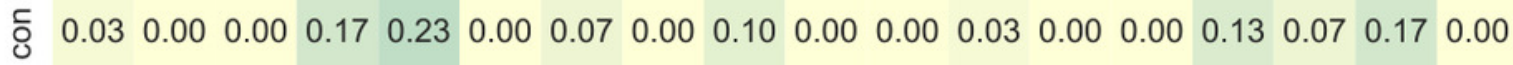
$\begin{array}{lllllllllllllllllll}\text { \& } & 0.00 & 0.07 & 0.08 & 0.03 & 0.09 & 0.27 & 0.06 & 0.17 & 0.00 & 0.02 & 0.06 & 0.01 & 0.01 & 0.00 & 0.04 & 0.03 & 0.07 & 0.00\end{array}$

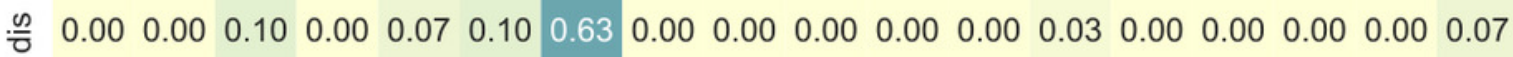

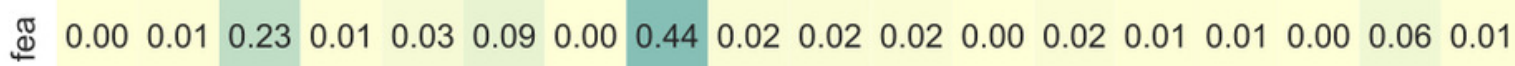

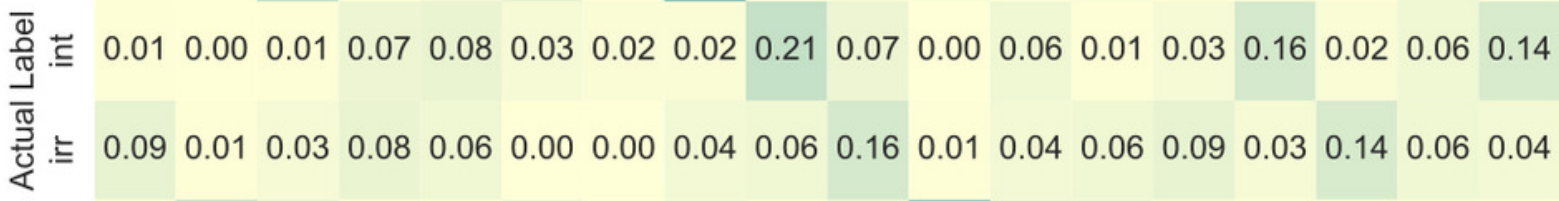

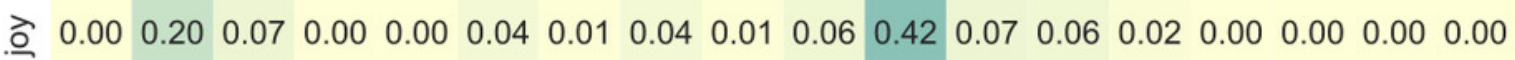
$\begin{array}{lllllllllllllllllll}\frac{\varrho}{\alpha} & 0.03 & 0.04 & 0.01 & 0.00 & 0.04 & 0.00 & 0.01 & 0.00 & 0.04 & 0.00 & 0.06 & 0.33 & 0.01 & 0.11 & 0.04 & 0.07 & 0.03 & 0.16\end{array}$

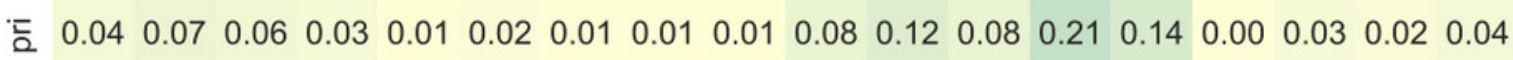
$\begin{array}{lllllllllllllllllll}\Phi & 0.06 & 0.00 & 0.00 & 0.02 & 0.02 & 0.01 & 0.01 & 0.00 & 0.02 & 0.07 & 0.01 & 0.16 & 0.03 & 0.38 & 0.03 & 0.10 & 0.07 & 0.01\end{array}$ ఫ్ఞ

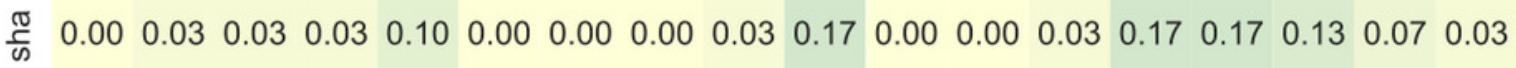

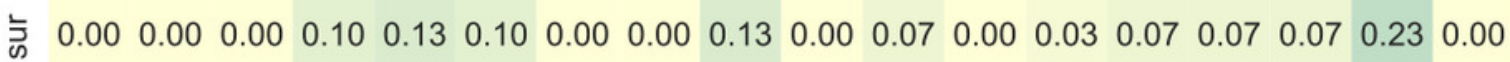

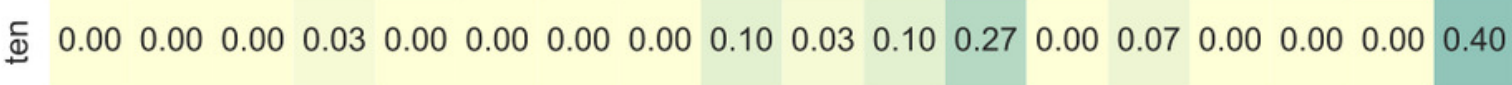
adm amu ang anx con des dis fea int irr joy ple pri rel sad sha sur ten Predicted Label 


\section{Figure 3}

Summary of the most important features (eGeMAPS) for classification of each emotion for the best performing audio classifier (Elastic Net).

See Figure $\mathrm{S} 7$ for a complete list that includes all features. Functionals: $\mathrm{M}=$ arithmetic mean, $C V=$ coefficient of variation, $\mathrm{P} 20=20^{\text {th }}$ percentile, $\mathrm{P} 80=80^{\text {th }}$ percentile, $\mathrm{P}$ range $=$ range of $20^{\text {th }}$ to $80^{\text {th }}$ percentile, M/SD rising slope $=$ mean/standard deviation of the slope of rising signal parts, M/SD falling slope $=$ mean/standard deviation of signal parts with falling slope. Features: voiced/unvoiced $=$ feature was based on voiced/unvoiced regions only; F0 $=$ logarithmic fundamental frequency (FO) on a semitone frequency scale; jitter $=$ deviations in individual consecutive F0 period lengths; F1/F2/F3 frequency/bandwidth $=$ centre frequency/bandwidth of the first, second, and third formants; loudness = estimate of perceived signal intensity from an auditory spectrum; harmonics to noise ratio $=$ relation of energy in harmonic components to energy in noise-like components; shimmer = difference of the peak amplitudes of consecutive F0 periods; equivalent sound level = logarithmic average sound pressure level; alpha ratio = ratio of the summed energy from $50-1000 \mathrm{~Hz}$ and 1-5 $\mathrm{kHz}$; Hammarberg index = ratio of the strongest energy peak in the $0-2 \mathrm{kHz}$ region to the strongest peak in the 2-5 kHz region; spectral slope 0-500/500-1500 = linear regression slope of the logarithmic power spectrum within the two given bands; spectral flux $=$ difference of the spectra of two consecutive frames; F1/F2/F3 amplitude $=$ first, second, and third formant relative energy; MFCC 1-4 = Mel-Frequency Cepstral Coefficients 1-4; loudness peaks per second $=$ number of loudness peaks per second; voiced segments per second $=$ number of continuous voiced regions per second; voiced segment length = length of continuous voiced regions. 


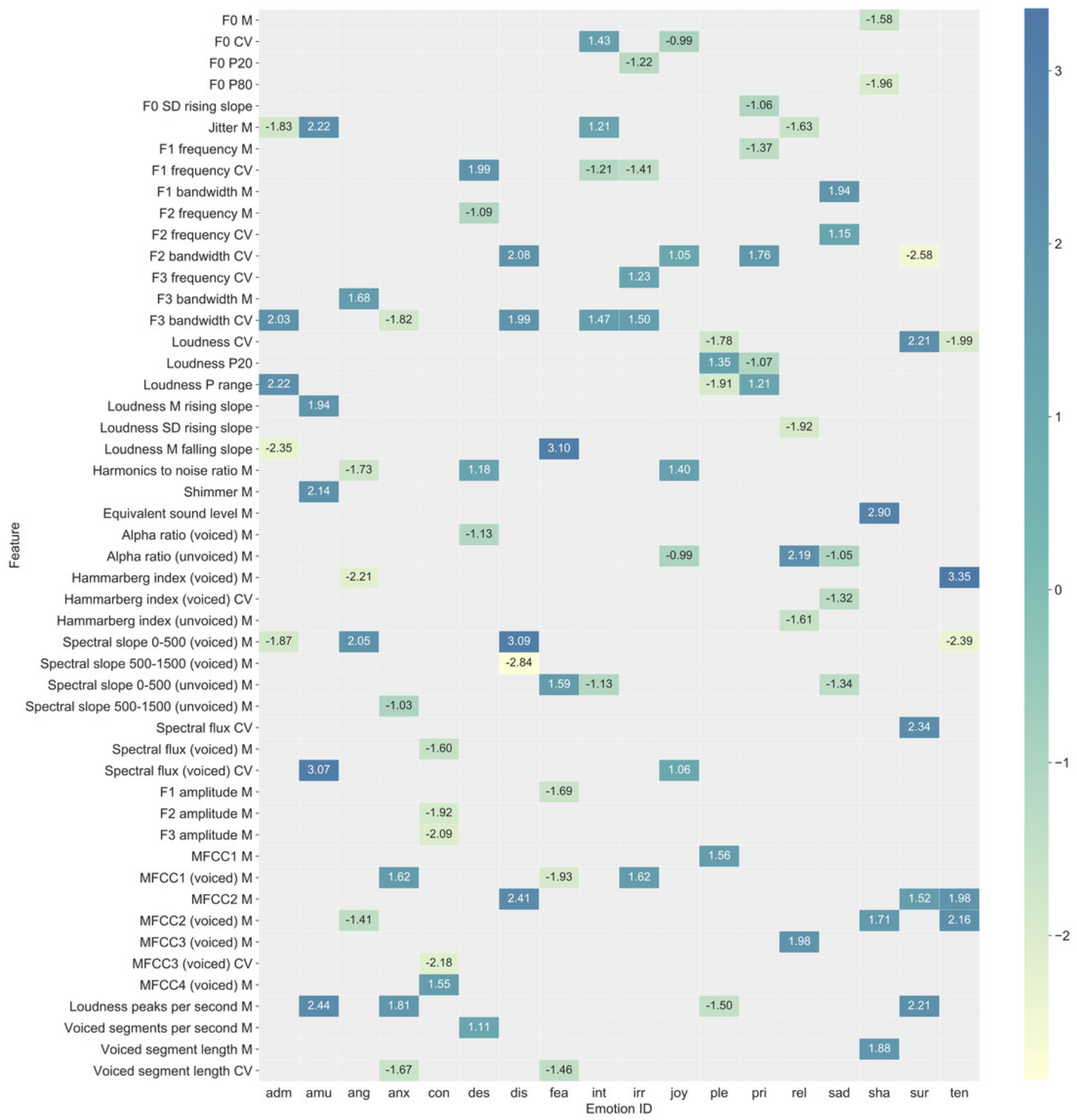


Figure 4

\section{Summary of the most important features (Facial Action Units and functionals) for classification of each emotion for the best performing video classifier (random forest).}

See Figure S8 for a complete list that includes all features. Functionals: $\mathrm{M}=$ arithmetic mean, $\mathrm{CV}=$ coefficient of variation, $\mathrm{P} 20=20^{\text {th }}$ percentile, $\mathrm{P} 50=50^{\text {th }}$ percentile, $\mathrm{P} 80=80^{\text {th }}$ percentile, $\mathrm{P}$ range $=$ range of $20^{\text {th }}$ to $80^{\text {th }}$ percentile, $\mathrm{N}$ peaks $=$ number of peaks .

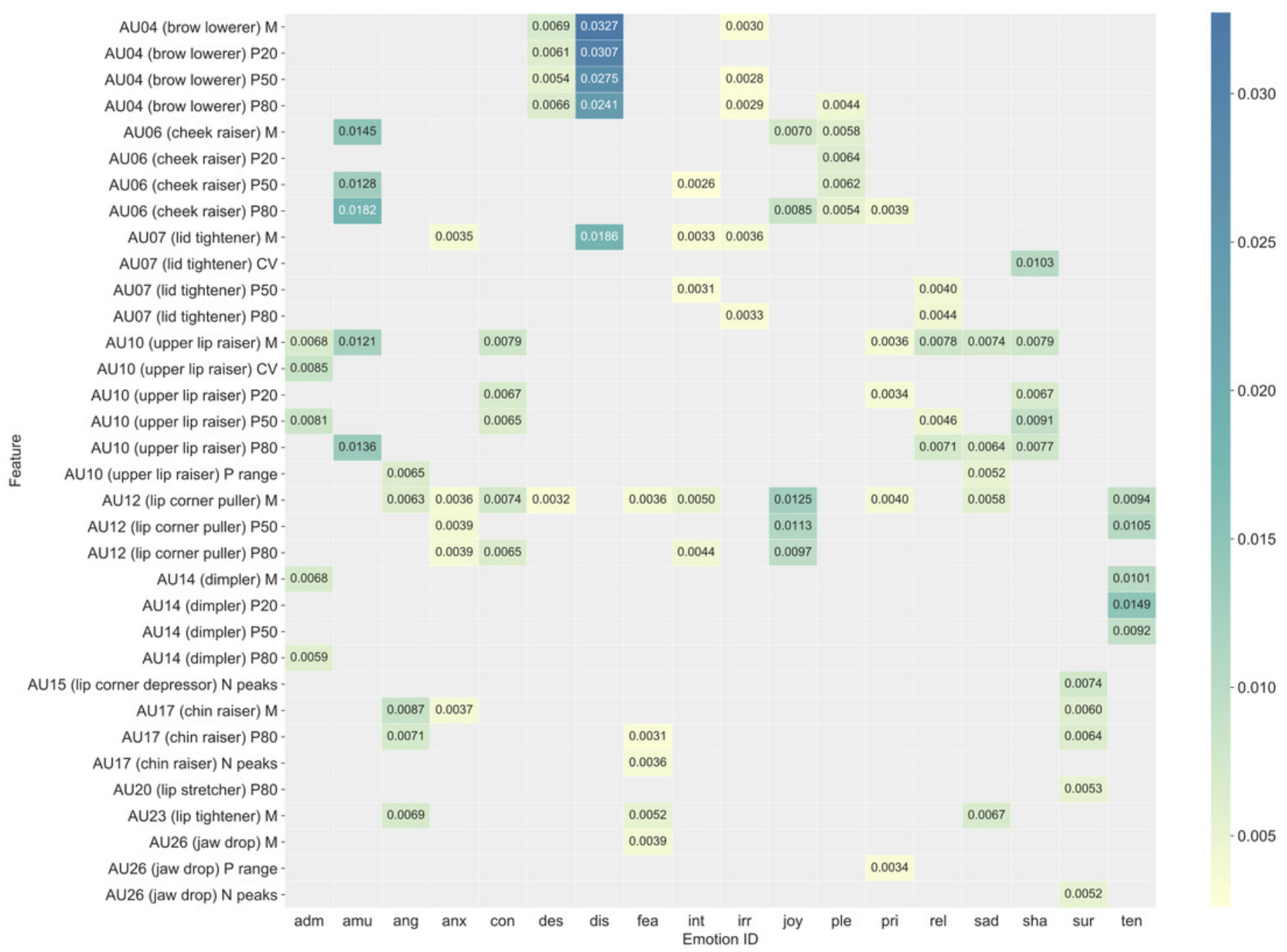




\section{Figure 5}

k-Means for $\mathrm{k}=2$ (top left) and hierarchical clustering for $\mathrm{k}=2$ and complete distance method (top right) before dimensionality reduction, with percentage of files per emotion and cluster.

The bottom two graphs show k-Means for $k=2$ (bottom left) and hierarchical clustering for $k$ $=2$ and weighted distance method (bottom right) after dimensionality reduction. $\mathrm{adm}=$ admiration, amu = amusement, ang = anger, anx = anxiety/worry, con = contempt, des = despair, dis $=$ disgust, fea $=$ panic fear, int $=$ interest, irr $=$ irritation, joy $=$ joy/elation, ple $=$ sensual pleasure, pri $=$ pride, rel $=$ relief, sad $=$ sadness, sha $=$ shame, sur $=$ surprise, ten $=$ tenderness.
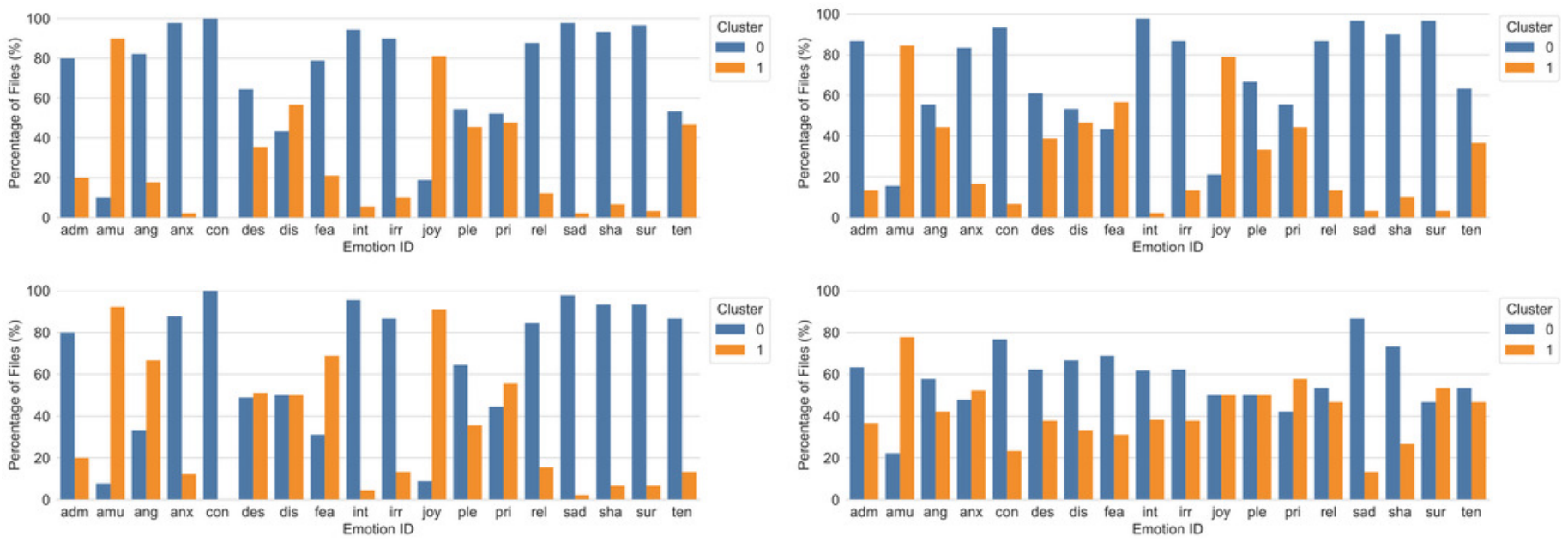
Figure 6

Fragment of the decision tree used to interpret the clustering.

The model was trained on the output of $k-M e a n s ~(k=2$; before dimensionality reduction).

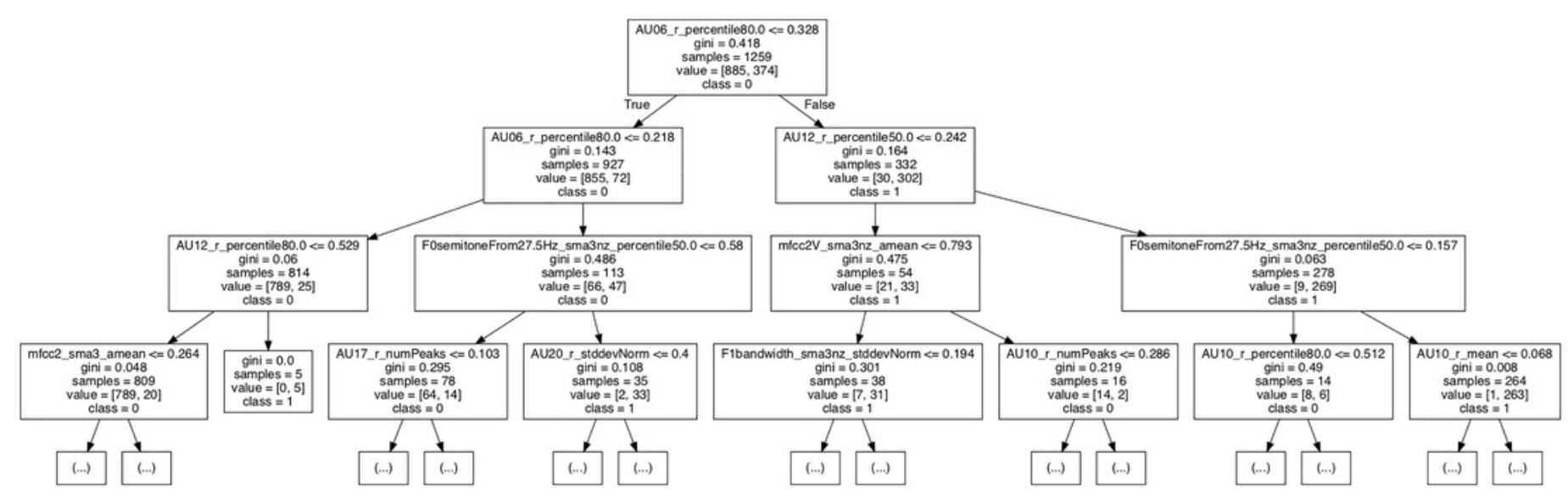




\section{Figure 7}

PCA 2D visualization of the multimodal dataset colored by emotion.

Note that 18 non-unique colors were used.

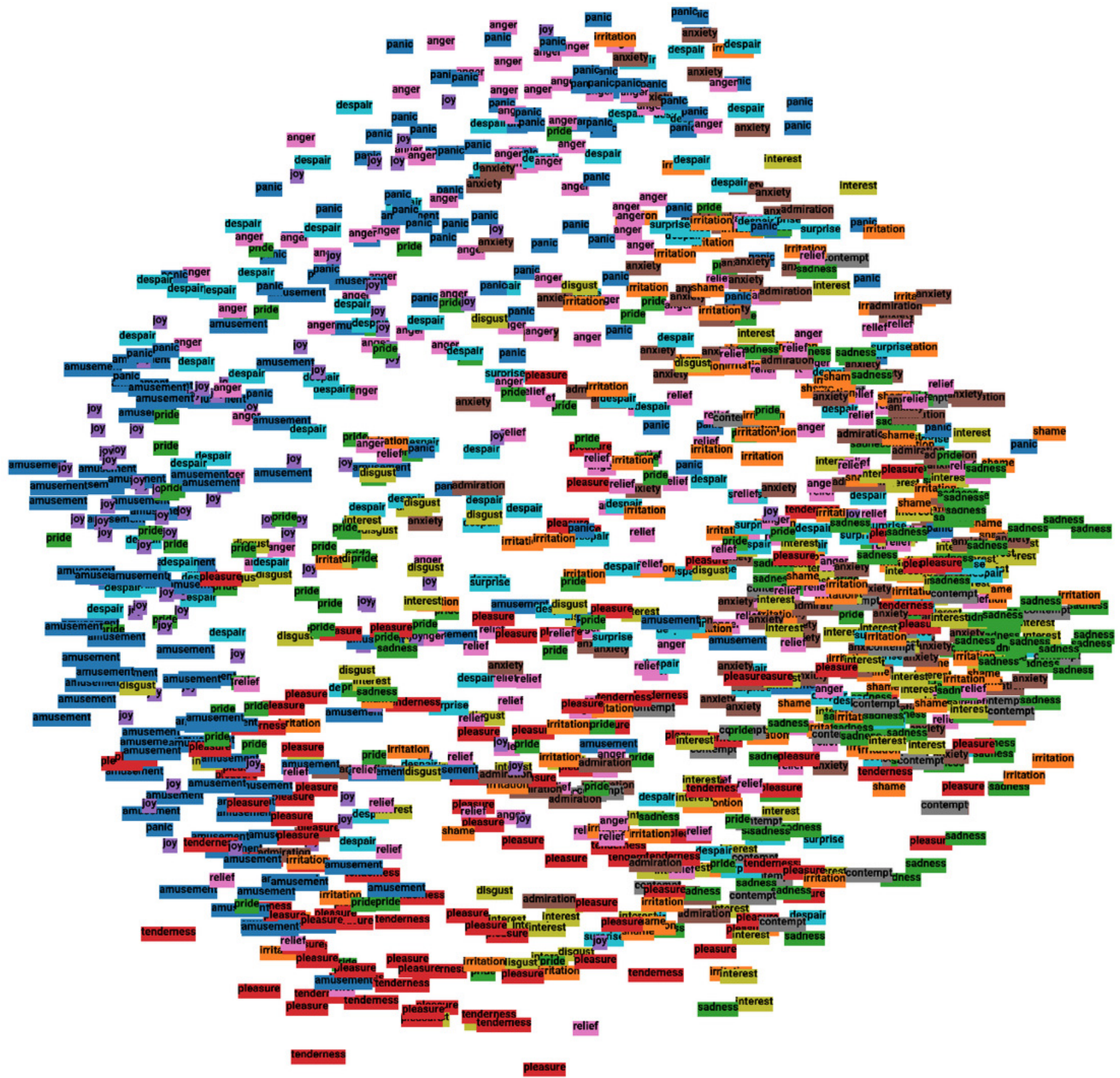


Figure 8

t-SNE 2D visualization of the multimodal dataset colored by emotion (a), valence (b), actor (c), and sex (d).
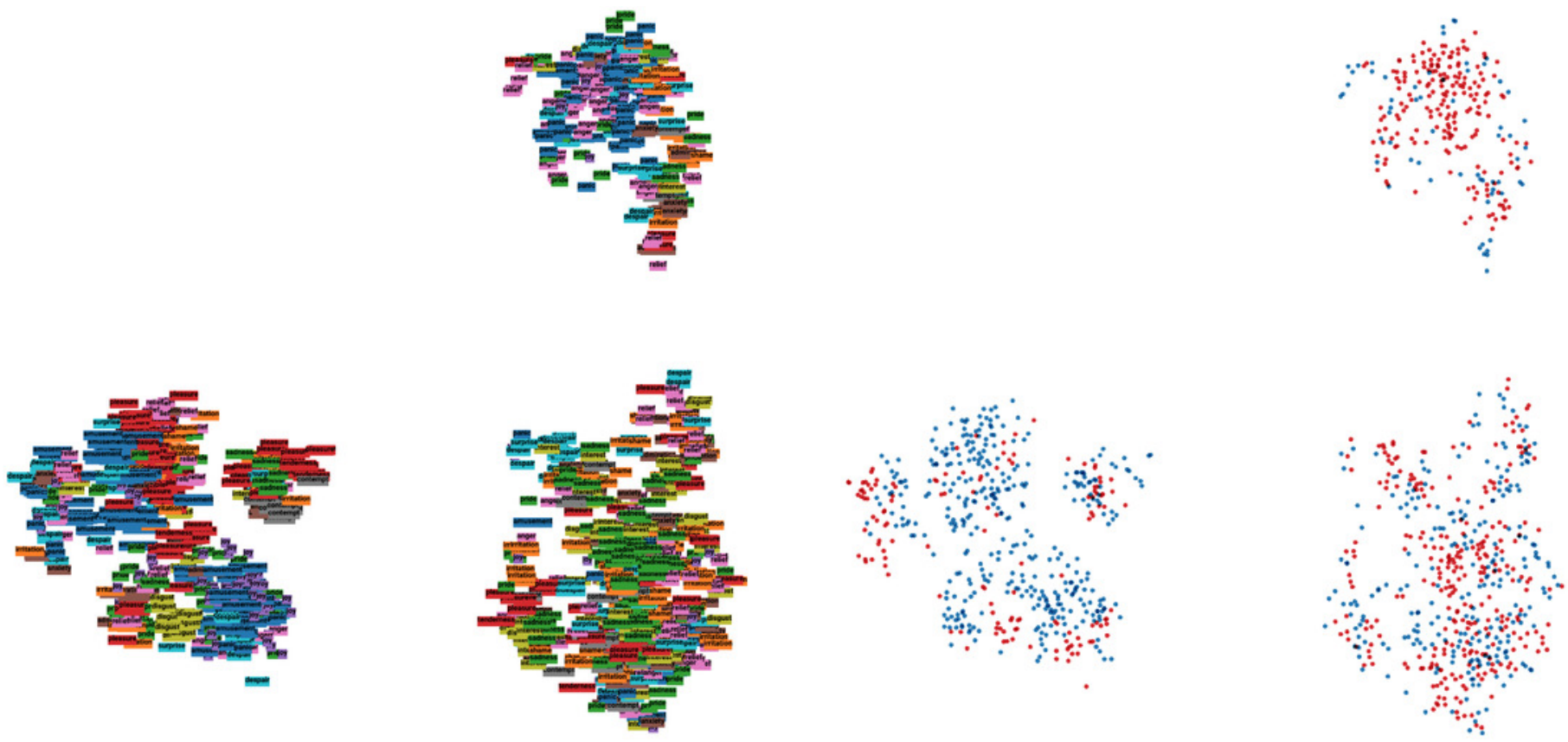

(a)

(b)
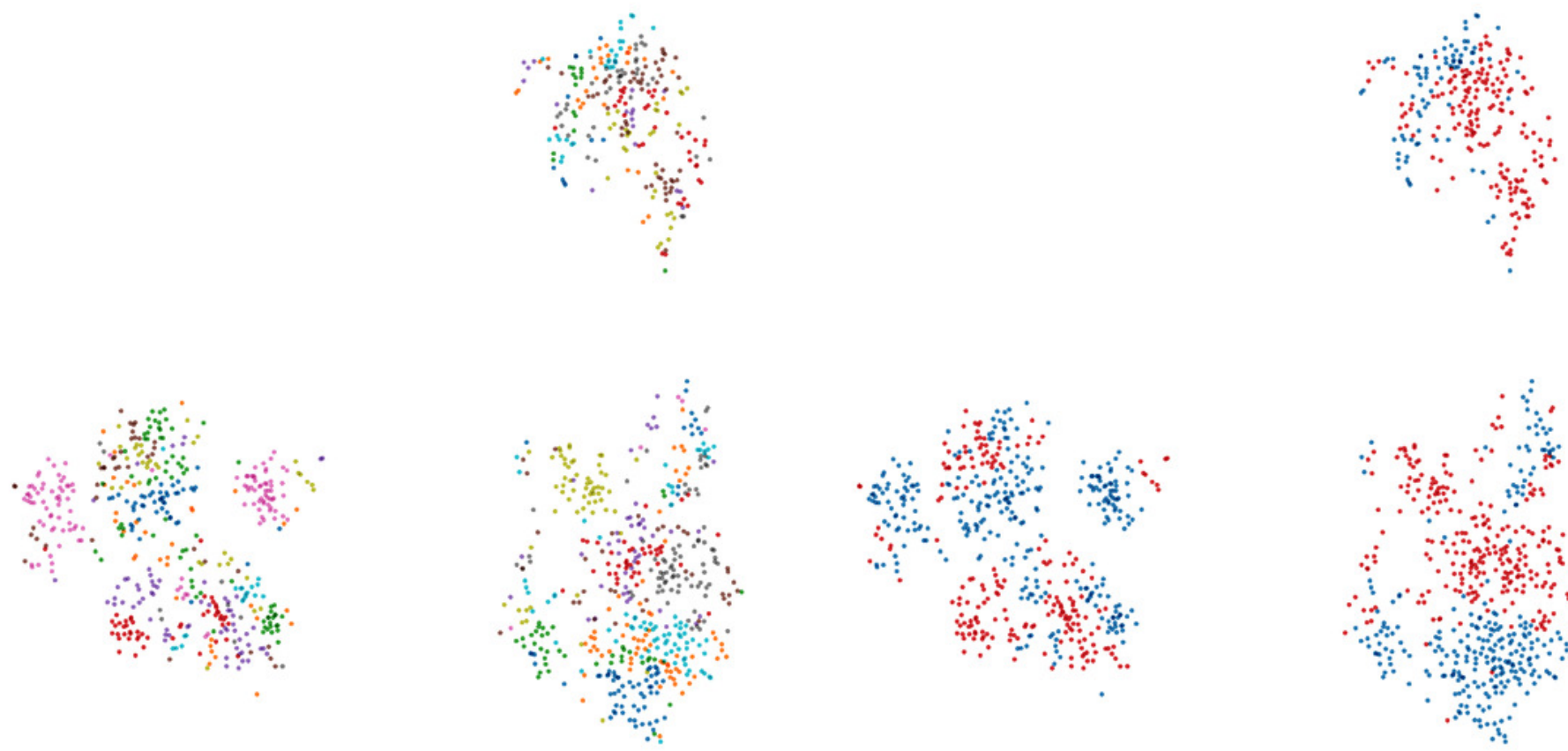

(c)

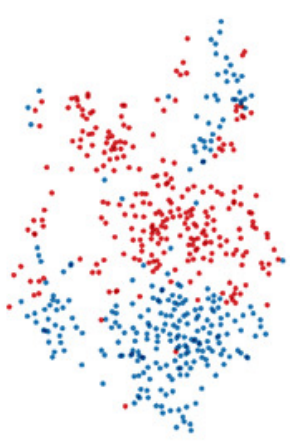

(d) 
Figure 9

UMAP 2D visualization of the multimodal dataset colored by emotion (a), valence (b), $\operatorname{actor}(\mathrm{c})$, and sex (d).

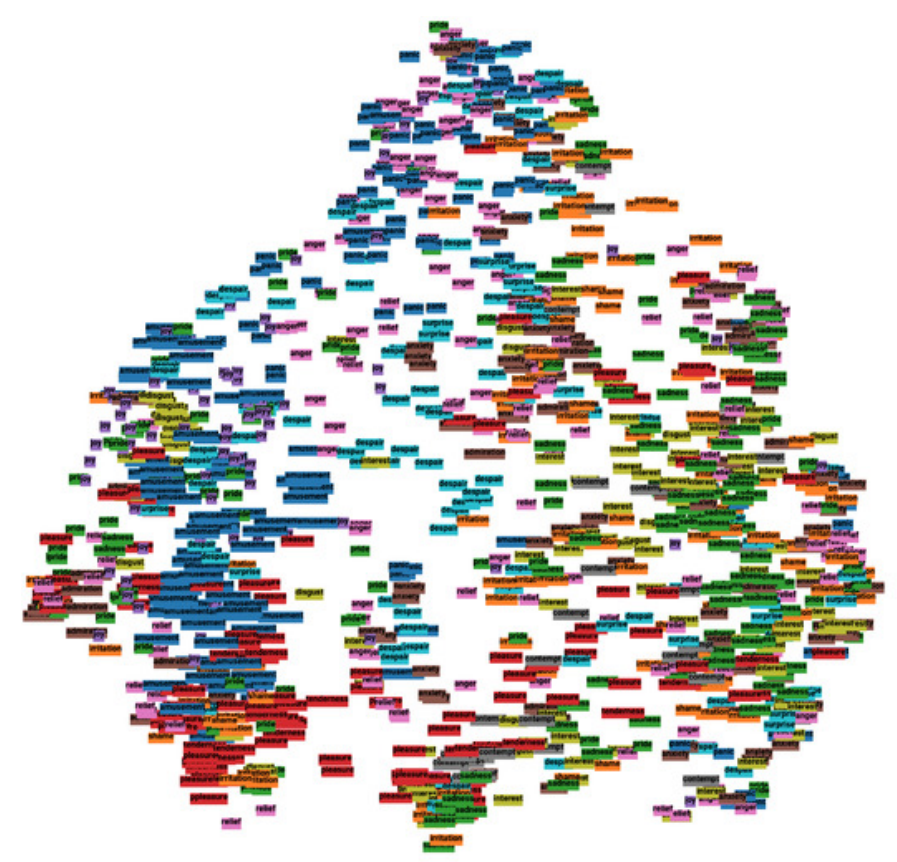

(a)

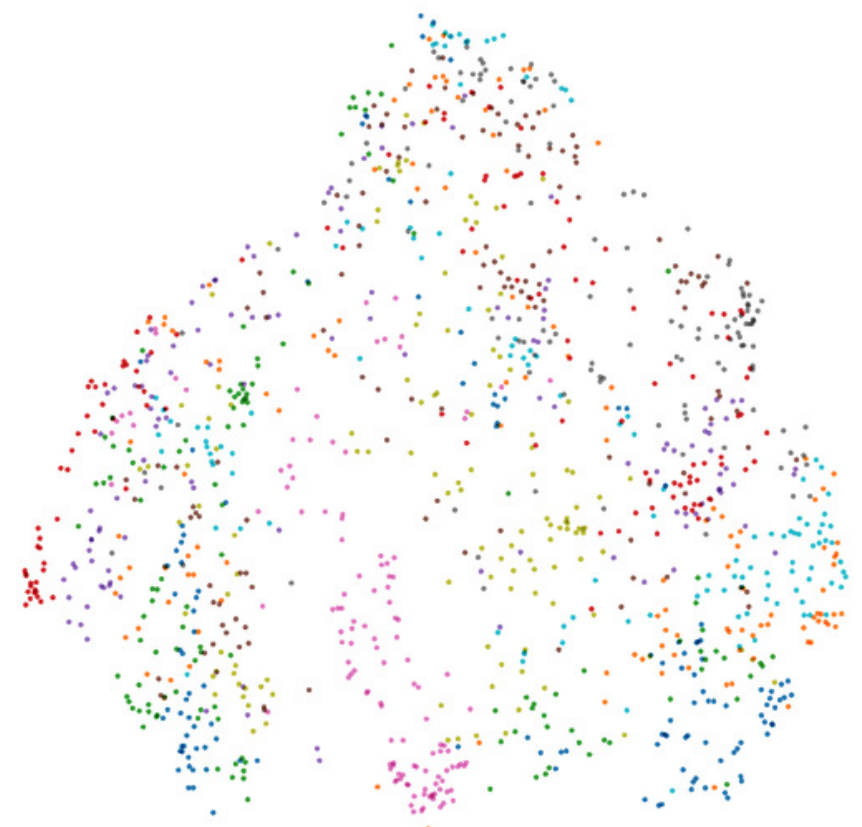

(c)

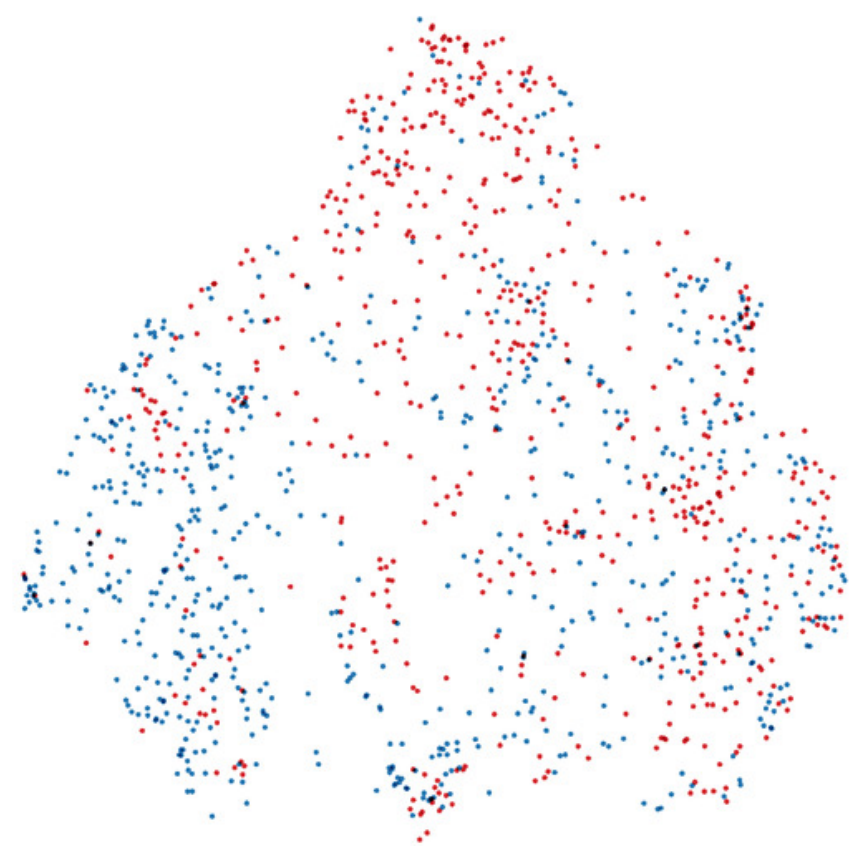

(b)

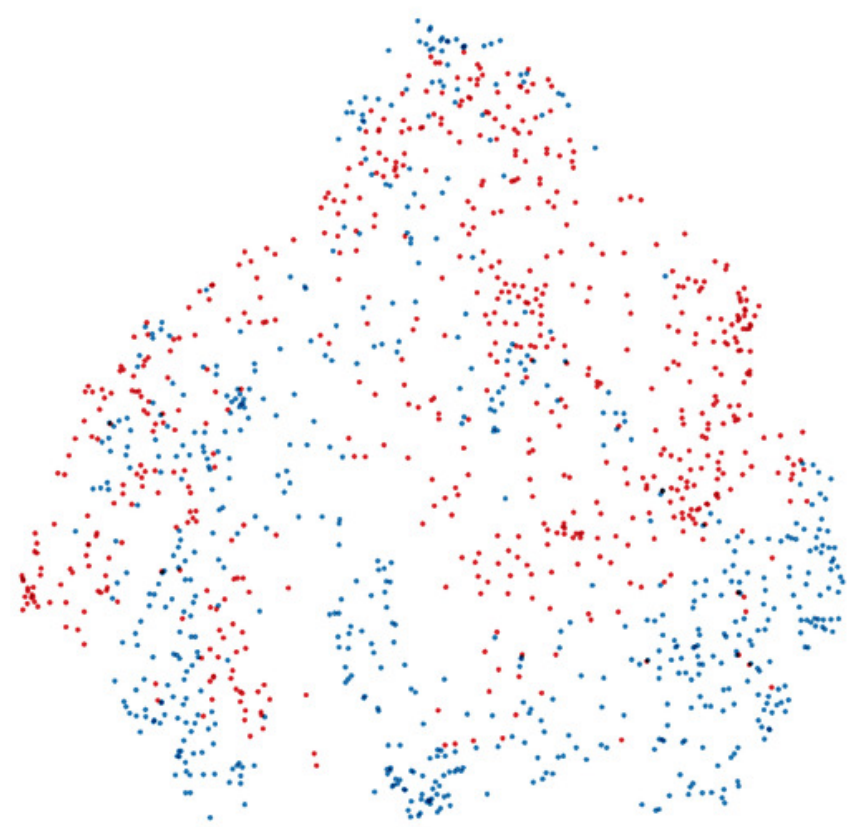

(d) 